\title{
CERTAIN CLASSES OF ANALYTIC FUNCTIONS OF TWO REAL VARIABLES AND THEIR PROPERTIES
}

\author{
BY \\ STEFAN BERGMAN
}

1. Introduction. Let

$$
\mathrm{L}(U) \equiv \Delta U / 4+A(\partial U / \partial x)+B(\partial U / \partial y)+C U=0
$$

be a partial differential equation whose coefficients $A, B, C$ are supposed to be entire functions of $x$ and $y$.

As was shown in previous papers, with every real solution $U(x, y)$ of (1.1) it is possible to associate another real solution $V(x, y)$ such that the class $\mathcal{C}$ of complex solutions $u=U+i V$ obtained in this way possesses many prcperties similar to those of analytic functions of a complex variable $\left({ }^{1}\right)$. For instance:

I. There exists a set of solutions $\left\{\phi_{\nu}\right\}, \phi_{\nu}(x, y)=U_{\nu}(x, y)+i V_{\nu}(x, y)$, $\mathrm{L}\left(\phi_{\nu}\right)=0$, each of which is an entire function of $x$ and $y$, which can be considered as an analogue of $\left\{(x+i y)^{\nu}\right\}$ :

i. $\left\{\phi_{\nu}\right\}$ behaves similarly to the powers for large values of $\nu$, more exactly, we have $\left|\phi_{\nu}(x, y)-H(x, y)(x+i y)^{\nu}\right| \leqq C_{1}(x, y) / 2(\nu+1)$ where $H$ and $C_{1}$ are entire functions which are independent of $\nu$.

ii. $c \phi_{\nu}, c$ an arbitrary complex constant, is again a member of class $\mathcal{C}$.

iii. Every solution $u$ which is regular in a circle $x^{2}+y^{2} \leqq \rho^{2}$ can be developed there in a uniformly convergent series, $u=\sum_{\nu=1}^{\infty} a_{\nu} \phi_{\nu}$ (see $[4, \S 6]$ ).

iv. Every $u$ which is regular in a simply connected (closed) domain $\overline{\mathfrak{B}}$, $(0,0) \in \mathfrak{B}$, can be approximated there by a linear combination, $\sum_{\nu=1}^{n} a_{\nu}^{(n)} \phi_{\nu}$, of the $\phi_{\nu}$ (an analogue of Runge's theorem). See $[4, \S 6]$.

II. There exist simple relations (similar to those of the theory of analytic functions of a complex variable) between the properties of a solution $u$ whose series development at the origin is $u=\sum_{m, n=0}^{\infty} A_{m n} z^{m} \bar{z}^{n}, z=x+i y, \bar{z}=x-i y$, and the behavior of the sequence $\left\{A_{n 0}\right\}, n=0,1,2,3, \ldots$. For example:

i. $u$ is regular in every domain in which $\sum_{m=0}^{\infty} A_{m 0} z^{m}$ is regular. (Note that consequently the location of singularities of $u$ is determined by the $A_{m 0}$, $m=0,1,2, \cdots$, independently of the coefficients of the equation.) $[4, \S 7]$.

ii. If $\sum_{m=0}^{\infty}\left|A_{m 0}\right|^{2}<\infty$ then $u=\sum_{m, n=0}^{\infty} A_{m n} z^{m} \bar{z}^{n}$ is regular in $x^{2}+y^{2}<1$ and possesses boundary values for radial paths of approach, almost everywhere. Again the set of points where the boundary values exist is independent of the coefficients of the equation (see $[4, \S 7]$ ).

Presented to the Society, April 29, 1944; received by the editors March 30, 1944.

(1) The conjugate $V$ is uniquely determined by $U$ within a fixed solution multiplied by an arbitrary constant. $V$ and $U$ are connected by certain linear integrodifferential relations (see $[4, \S 8])$. The numbers in brackets refer to the bibliography at the end of the paper. The present paper does not presuppose knowledge of previous publications. 
Clearly the results obtained about these (complex) solutions $u$ yield corresponding results about the real solution $U$, and therefore the introduction of the class of complex solutions indicated above represents a powerful tool in the theory of partial differential equations.

The study of the duality which exists between the theory of (complex) solutions $u$, mentioned above, and that of analytic functions of a complex variable is essentially based on the fact that the solutions $u$ can be obtained from analytic functions $f(z)$ by means of an integral operator.

$$
\begin{aligned}
u(z, \bar{z}) & =\mathrm{P}(f) \equiv \int_{-1}^{1} \mathrm{E}(z, \bar{z}, t) f\left(z\left(1-t^{2}\right) / 2\right) d t /\left(1-t^{2}\right)^{1 / 2}, \\
z & =x+i y, \quad \bar{z}=x-i y, \\
f(z / 2) & =\frac{2}{\pi} \int_{0}^{\pi / 2} z \sin \theta \frac{\partial u\left(z \sin ^{2} \theta, 0\right)}{\partial\left(z \sin ^{2} \theta\right)} d \theta+\frac{1}{\pi} u(0,0) .
\end{aligned}
$$

Here $\mathbf{E}$ is a conveniently chosen entire function of $z$ and $\bar{z}$ (which depends only upon the coefficients $A, B, C$ of the equation $\mathrm{L}$ ). When $f$ ranges over the totality of analytic functions of a complex variable, $\operatorname{Re} u$ ranges over the totality of real solutions of (1.1). ( $f$ and $u$ are supposed to be regular at the origin.) See [3].

Remark. We note that the operator $\mathrm{P}$ is not defined only with respect to some particular domain of the $x y$-plane; it can be shown that both functions $u(z, \bar{z}), \bar{z}$ conjugate to $z$, and $f(z / 2)$ are regular in the same domain of the $x y$-plane, and that the representation (1.2) is valid in every simply connected domain $\mathfrak{F},(0,0) \in \mathfrak{F}$, in which $u(z, \bar{z})$ is regular.

Definition. The totality of functions $u$, which we obtain by means of an operator (1.2) when $f$ ranges over the class of analytic functions of a complex variable, is denoted as the class $\mathcal{C}(\mathbf{E}) ; \mathbf{E}$ is called the generating function of the class $\mathcal{C}(\mathrm{E})$.

The relation (1.2) can be interpreted as a mapping, in function space, of the class of analytic functions of one complex variable into the class of functions $\mathcal{C}(\mathbf{E})$. A more thorough analysis shows that the above-mentioned method of investigation which is based on the study of the integral operators of the type (1.2) is not limited to the case where the transformed functions $u$ satisfy equation $(1.1)$ (see $[4, \$ 2])$.

The two following properties of the set of functions are sufficient in order to establish the duality between the theory of functions $u$ and that of one complex variable.

i. Every function $u(x+i y, x-i y)$ which is regular in a domain $\mathfrak{B}$ of the (real) $x y$-plane can be analytically continued for complex values of $x$ and $y$ into a certain (four-dimensional) domain $\Re(\mathscr{B})$ which depends on $\mathscr{B}$ but is independent of $u$, and it is possible to represent $u$ in $\Re(\mathfrak{B})$ in terms of values of $u$ and its derivatives on the boundary $\mathfrak{b}$ of $\mathfrak{B}$. 
ii. The generating function $\mathbf{E}$ is of the first kind, that is, has the form

$$
\mathrm{E}=\exp \left(-\int_{0}^{\bar{z}} a d \bar{z}\right)\left[1+z \bar{z} t^{2} \mathrm{E}^{*}(z, \bar{z}, t)\right]
$$

where $\mathrm{E}^{*}$ is also an entire function $\left(^{2}\right)$ of $z, \bar{z}$. (Classes of functions of this kind occur in other fields as well $\left({ }^{3}\right)$, for example, in the theory of linear partial differential equations of order $2 n, n>1$.)

In this connection in [4] we considered what types of results of the theory of analytic functions can be carried over to the theory of functions which satisfy the conditions $i$ and ii (above).

On the other hand one may obtain complex solutions of (1.1) using operators which differ from those described immediately above. One again obtains classes of functions $(U+i W)$ where $U$ ranges over the totality of (real) solutions of (1.1) and $W$ is determined in a unique way (within a constant multiplied by a fixed function) from $U$ by some suitable linear relation.

A particular case of this question is the investigation of classes which can be obtained by an integral operator of the form (1.2) or similar to (1.2) but where $\mathbf{E}$ is not necessarily a generating function of the first kind. (Clearly the conjugate $W$ corresponding to a given real solution $U$ changes if we pass from one generating function $\mathbf{E}$ to another generating function.)

One of the motivations of the study of the preceding question is the fact that in certain investigations of real solutions $U$, these functions $U+i W$ ( $W$ obtained as described immediately above) would be more suitable than those obtained by means of the operator (1.2) with the generating function of first kind $\left.{ }^{4}\right)$. The first step in this direction is to determine all operators which transform analytic functions into solutions of differential equations, or at least a large class of such operators $\left(^{5}\right)$.

The first part of the present paper is devoted to this question.

(2) One of the essential results of paper [3] was the proof that to every equation (1.1) there exists a class of complex solutions $u$ satisfying $i$, and that the $\operatorname{Re} u$ represents the totality of (real) solutions of (1.1) each of which is regular at the origin.

(3) We wish, further, to stress the fact the results obtained for linear partial differential equations are of interest for many nonlinear equations of physics. By suitable transformations the (nonlinear) equations of the theory of a compressible fluid, of plasticity, of the theory of heat can, in many instances, be reduced to linear equations, of the type considered in this approach.

(4) Indeed, such a situation occurs in the study of growth of entire solutions $U(x, y)$. It is possible to show that for various differential equations (1.1) there exists a generating function $\mathrm{E}=Q$ exp $P$, where $Q$ and $P$ are polynomials in $t$, whose coefficients are algebraic functions of the coefficients of $\mathrm{L}$, and it is of ten much easier to determine the growth of $Q \exp P$ than the corresponding generating function of the first kind (see [5]). Another such case occurs in the study of singularities of solutions of (1.1).

(5) Another problem of the theory, which however has been almost unexplored until the present, is the study of different forms of the same operator and the connections between different classes of functions. 
In $\S \S 2$ and 3 we determine a large class of integral operators which transform the totality of analytic functions of one complex variable into (certain) complex solutions of (1.1).

Simultaneously in $\$ 2$ we attempt to show that the transition from real solutions $U$ to the functions $u \in \mathcal{C}(\mathbf{E}), \mathbf{E}$ being a generating function of the first kind, can be considered as a natural generalization of the transition from a harmonic function of two variables to the analytic function of a complex variable of which it is the real part. We show, namely, that certain requirements (which are fulfilled in the case of harmonic functions) entail that the operation transforming analytic functions into real solutions of $U$ must be representable as the real part of (1.2), $\mathbf{E}$ being a generating function of the first kind, or derivable in a simple way from such an operator.

The second part of the present paper is devoted to the study of singularities of complex solutions of (1.1).

In $\S 5$, we show that in the case of classes of (complex) solutions with generating functions of the first kind singularities exist which are similar to branch points and poles $\left({ }^{6}\right)$.

In $\S \S 6-8$ we study the behaviour at infinity of entire functions of the class $\mathcal{C}(\mathbf{E})$ where $\mathbf{E}$ satisfies certain conditions but is not necessarily a generating function of the first kind. The following result is derived there. Let $n\left[r,(u-b)^{-1}\right]$ denote the sum of the "indices" of points which lie in the circle $x^{2}+y^{2} \leqq r^{2}$ and where $u$ assumes the value $b$. We then give an upper bound for the growth of $n\left[r,(u-b)^{-1}\right], r \rightarrow \infty$, in terms of the $A_{m 0}, m=0,1,2, \cdots$, $\sum \sum A_{m n} z^{m} \bar{z}^{n}, z=x+i y, \bar{z}=x-i y$, being the series development of $u$ at the origin.

\section{INTEGRAL OPERATORS IN THE THEORY OF PARTIAL DIFFERENTIAL EQUATIONS}

As indicated in the introduction, in this chapter a large class of integral operators which transform analytic functions into complex solutions of (1.1) will be determined and an attempt will be made to derive the integral representation (1.2), with generating function $\mathbf{E}$ of the first kind, as a natural generalization of the representation of harmonic functions in terms of analytic functions.

2. The associate function. Every real( $\left.{ }^{7}\right)$ function $\phi(x, y)=U(z, \bar{z})$, $z=x+i y, \bar{z}=x-i y$, of two real variables $x$ and $y$ may be written in the form

$$
\phi(x, y)=U(z, \bar{z})=[k(z, \bar{z})+\bar{k}(\bar{z}, z)] / 2=\operatorname{Re}[k(z, \bar{z})], \operatorname{Re}=\text { Real part, }
$$

where $k(z, \bar{z})$ is some complex function and $\bar{k}(\bar{z}, z)$ is the conjugate of $k(z, \bar{z})$.

${ }^{(6)}$ It should, however, be stressed that while the branch points have the same character as those in the theory of analytic functions, the singularities which correspond to the poles are no longer single-valued, but represent a combination of a pole and a logarithmic branch point.

$\left(^{7}\right)$ Real $=$ real valued for real values of $x$ and $y$. If $U(z, \bar{z})=\sum A_{m n} z^{m} \bar{z}^{n}$, then $A_{m n}=\overline{A_{n m}}$ is the necessary and sufficient condition that $U(z, \bar{z})$ be real. 
In fact we may choose for $k(z, \bar{z})$ any function of the form $U(z, \bar{z})+i V(z, \bar{z})$, where $V(z, \bar{z})$ is an arbitrary real function of $z$ and $\bar{z}$.

If $\phi$ is a harmonic function, then for $k$ we may choose an analytic function of a complex variable $z$, say $f(z)$. In this case if we continue $\phi(x, y)$ to complex values of the arguments, we obtain very simple relations

$$
k(z, 0)=f(z)=2 U(z, 0)+\text { const., } k(0, \bar{z})=f(0)=\text { const. }
$$

In many instances the study of the analytic function $f(z)$ leads to interesting results about the corresponding harmonic function. Thus, the introduction of analytic functions provides a powerful tool in the theory of harmonic functions.

Consider, now, a partial differential equation of the elliptic type

$$
\begin{gathered}
\mathrm{L}(U) \equiv \Delta U / 4+A U_{x}+B U_{y}+C U \equiv U_{z \bar{z}}+a U_{z}+\bar{a} U_{\bar{z}}+c U=0, \\
a=A+i B, \quad \bar{a}=A-i B, \quad C=c, \\
U_{z}=2^{-1}\left(U_{x}-U_{y}\right), \quad U_{x}=\partial U / \partial x, \cdots .
\end{gathered}
$$

Here $A, B$, and $C$ are functions of the real variables $x$ and $y$ which when continued to complex values of the arguments are supposed to be analytic functions of two complex variables, $x$ and $y$, which are regular in a domain $\mathfrak{D}$ containing the origin of the (four-dimensional) space of these two complex variables. ( $A, B, C$ are supposed to be real, see footnote 7.) In order to investigate the functions satisfying (2.3) in the real $x y$-plane, one may attempt (by analogy with the theory of harmonic functions) to generalize the abovementioned method of attack, which method is based on the use of functions of a complex variable. This may be done by associating with every function satisfying (2.3) an analytic function of a complex variable.

In certain respects it is more convenient to introduce operators which transform an analytic function of a complex variable into a complex solution $u=U+i V$, whose real part is $U$ and whose imaginary part $V$ is uniquely determined by $\left(^{8}\right) U$ (to within a constant multiplied by a fixed function).

Under rather general assumptions an operator which links $u(z, \bar{z})$ to $f(z)$ can be defined in the following manner:

Let $u(z, \bar{z})=\sum_{m=0}^{\infty} \sum_{n=0}^{\infty} A_{m n} z^{m} \bar{z}^{n}$ and $f(z)=\sum_{n=0}^{\infty} a_{n} z^{n}$. Substituting $\bar{z}=0$ we obtain

$$
u(z, 0)=\sum_{m=0}^{\infty} A_{m 0} z^{m}
$$

Let

$$
\left\{\tau_{m n}\right\}, \quad m=0,1,2, \cdots ; n=0,1,2, \cdots,
$$

(8) In $\$ 4$ we shall discuss in more detail several possible choices of $V$, in particular also the case where $V$ is not necessarily a solution of (2.3). 
be an infinite matrix. Writing

$$
\sum_{n=0}^{\infty}\left(\sum_{m=0}^{\infty} \tau_{m n} a_{m}\right) z^{n}
$$

and making some assumptions about the convergence of (2.6) we obtain from $f$ another function of a complex variable. Since $u$ is a solution of (2.3) it is uniquely determined if the $A_{m 0}$ and $A_{0 m}$ are prescribed. We may thus define an operator which transforms $f$ into $u(z, \bar{z})$ by the introduction of the matrix (2.5), setting

$$
\sum_{m=0}^{\infty} \tau_{m n} a_{m}=A_{n 0}
$$

and prescribing the $A_{0 m}$ in a certain manner which depends only on $f(0)$ but is otherwise independent of $f$. For the sake of brevity we shall call $f(z)$ the associate of $U$ of the type $\left\{\tau_{m n}\right\}$.

Before proceeding further we wish to make a remark concerning the choice of the $\tau_{m n}$.

Consider $U(z, \bar{z})$ in the complex domain $\left({ }^{9}\right)$. The characteristics of (2.3) are $z=$ const. and $\bar{z}=$ const. If one wishes to associate with $U(z, \bar{z})$ an analytic function $f(z)$ which possesses the property that $f(z)$ and $U(z, \bar{z}), \bar{z}$ conjugate to $z$, have the same regularity domain in the (real) $x y$-plane, it is natural to choose $f(z)=2 U(z, 0)$, or some function which is in some simple way connected with $U(z, 0)$. Clearly, it is of importance that, in addition to this, both the operator which transforms $f$ into $U$ and the inverse operator be not too complicated, and further, that they possess many properties in common with harmonic functions. In this connection the following result was obtained in [4]:

There exists for every equation (2.3) an operator $S[f(z)]=k(z, \bar{z})$, defined for all functions $f(z)$ analytic at $z=0$, which transforms each of these functions into a function, $k(z, \bar{z})$, analytic at the origin. This operator $\mathrm{S}$ has a unique inverse which depends only on $\mathrm{L}$ (that is, on $A, B, C$ ) and not on the domain in which $k$ is considered. In terms of this operator every solution of (2.3) can be represented in the form $\left({ }^{10}\right)$

(9) Our aim is to obtain results concerning the behavior of $U(z, \bar{z})$ in the real domain, that is, when $z$ and $\bar{z}$ are conjugate to each other. The continuation in the complex domain is only a means of investigation.

In what follows, in most cases it will be clear whether we are considering $\phi(x, y) \equiv U(z, \bar{z})$ in the real domain, or whether we consider its analytic continuation in the space of two complex variables $x=x_{1}+i x_{2}, y=y_{1}+i y_{2}$. In the first case $z$ and $\bar{z}$ will be conjugate to each other; in the second case $z$ and $\bar{z}$ will be two independent complex variables. Sometimes, in doubtful cases, in order to indicate whether we are considering $U$ in the real or in the complex domain, we shall add after $U$ : " $z$ conjugate to $\bar{z}$ " or " $z$, $\bar{z}$ independent."

(10) The function $k(z, \bar{z})$ satisfies (in analogy to the case of harmonic functions) relation (2.2). 


$$
\begin{gathered}
U(z, \bar{z})=[p(z, \bar{z}) k(z, \bar{z})+p(\bar{z}, z) \bar{k}(\bar{z}, z)] / 2, \quad k(z, \bar{z})=\mathrm{S}[f(z)], \\
\bar{k}(\bar{z}, z)=\overline{k(z, \bar{z}) .}
\end{gathered}
$$

Here $p(z, \bar{z})$ is a function which depends only on $\mathrm{L}$ and has the property

$$
p(z, 0)=1 \text {. }
$$

The matrix $\left\{\tau_{m n}\right\}$ and $\sum_{m=0}^{\infty} A_{0 m} \bar{z}^{m}$ for the above operator are

$$
\begin{aligned}
\tau_{m m} & =\frac{\pi^{1 / 2} \Gamma(m+1 / 2)}{2^{m} \Gamma(m+1)}, \quad \tau_{m n}=0, \text { for } m \neq n, \\
\sum_{m=0}^{\infty} A_{0 m} \bar{z}^{m}=\exp \left[-\int_{0}^{\bar{z}} \bar{a}(\bar{z}, 0) d \bar{z}\right] . &
\end{aligned}
$$

In order to employ the operators in the theory of partial differential equations successfully, it is necessary to represent them in a form more convenient for applications than infinite matrices. One form is an integral representation.

An integral representation (1.2) for the above operator was obtained in [3], see also p. 311 of the present paper.

As mentioned in the introduction, it is in some instances preferable to use other operators than those previously introduced. In this connection the problem arises of finding all integral operators which transform analytic functions into complex solutions of (1.1), and determining their properties.

This problem will be partially solved in $\$ 3$ where a very large class of integral operators which transform analytic functions into complex solutions of (2.1) is determined, and the corresponding matrices $\left\{\tau_{m n}\right\}$ and the $A_{0 m}$, $m=0,1,2, \cdots$, are computed.

3. Integral representations for complex solutions of (2.3) in terms of analytic functions of a complex variable. As was pointed out at the end of $\$ 2$ the development of the described approach in the theory of partial differential equations requires finding for the operator $P$ which transforms the associate $f(z)$ in $p(z, \bar{z}) k(z, \bar{z})$ a form suitable for application.

The expression $\left({ }^{11}\right)$

$$
\mathrm{K}(z, \bar{z}) f(z)+\int \mathrm{E}(z, \bar{z}, t) f[n(z, t)] d t,
$$

where the integration is taken over $\mathfrak{i}^{1}$, represents a form for the operation $\mathrm{P}$ which is convenient for many purposes.

(11) This form of operator was suggested by the study of the equation $\Delta u+u=0$, where we have chosen $\mathrm{K}=0, \mathbf{E}=\left(1-t^{2}\right)^{-1 / 2}\left[\exp \left(i t(z \bar{z})^{1 / 2}\right)\right], n=z\left(1-t^{2}\right) / 2$, and $\mathfrak{i}^{1}=E[-1<t<1]$. Substituting $f(\zeta)=\zeta^{\nu}, z=r e^{i \phi}$ we then have $2^{-\nu} r^{\nu} e^{i \nu \phi} \int_{-1}^{1}[\exp (i t r)]\left(1-t^{2}\right)^{\nu-1 / 2} d t=\pi^{1 / 2} e^{i \nu \phi} \Gamma(\nu+1 / 2) J_{\nu}(r)$, where $J_{\nu}(r)$ are the Bessel functions of the $\nu$ th order. See [2, p. 390]. The analogy suggests the generalization of various results in the theory of Bessel functions to the case of operators of the form (3.1). By $E[\cdots]$ we denote the set of points whose coordinates satisfy the relations indicated in brackets. 
Here $\mathrm{K}(z, \bar{z}), \mathbf{E}(z, \bar{z}, t), n(z, t)$ are some fixed functions which are defined in the neighborhood $\mathfrak{u}^{4}$ of the origin, $z=\bar{z}=0$, and for $t \in \mathfrak{i}^{1}$. They are assumed to be differentiable with respect to $z, \bar{z}$ and $t$ as many times as will be necessary in our further considerations. The integration curve $i^{1}$ can be assumed either to be an open curve $t=t(\tau),-\pi<\tau<\pi, t(-\pi)=a, t(\pi)=b$ in the complex $t$-plane, or a closed curve.

The associate $f$ ranges over the totality of analytic functions of a complex variable. It is assumed to be regular for all points $n=n(z, t), t \in \mathfrak{i}^{1},|z|<\rho$, $\rho>0$ sufficiently small.

We shall denote by $\mathcal{C}=\mathcal{C}\left[\mathrm{E}, \mathrm{K}, n ; \mathfrak{i}^{1}\right]$ the class of functions of the form (3.1) which we obtain if $f$ ranges over the above indicated totality of functions.

Two questions arise:

1. How to determine $\mathbf{E}, \mathrm{K}, n, \mathfrak{i}^{1}$ in order that the functions of the obtained class are solutions of a given differential equation, $\mathrm{L}(u)=0$.

2. How to characterize the obtained totality of complex solutions of $\mathrm{L}(u)=0$ independently of the operator.

1. We consider at first the case where $i^{1}$ is an open curve.

Two simplifications can be made.

I. Since $\int_{a}^{b} \mathrm{E}(z, \bar{z}, t) f[n(z, t)] d t=\int_{-\pi}^{\pi}[\mathrm{E}(z, \bar{z}, t(\tau))] t^{\prime}(\tau) f[n(z, t(\tau))] d \tau$, we may assume from the start on that $\mathfrak{i}^{1}=E[-\pi<t<\pi]$.

II. If $p(z, \bar{z}) k(z, \bar{z})$ satisfies $(2.3)$ then $u(z, \bar{z})=\left[\exp \left(\int_{0}^{\bar{z}} a d \bar{z}\right)\right] p(z, \bar{z}) k(z, \bar{z})$ satisfies the equation

$$
\mathrm{L}^{*}(u) \equiv u_{z \bar{z}}+D u_{\bar{z}}+F u=0
$$

where $D=\bar{a}-\int_{0}^{\bar{z}} a_{z} d \bar{z}, F=-a_{z}-a \bar{a}+c$. (See $[3, \mathrm{p} .1172]$.) We assume therefore from the start that equation (2.3) has the form (3.2). We note that in this section for the sake of simplicity we shall omit the asterisk and write $\mathrm{L}$ in stead of $L^{*}$.

We notice that

and therefore

$$
\frac{\partial f[n(z, t)]}{\partial z}=\frac{\partial f[n(z, t)]}{\partial t} \cdot \frac{n_{z}}{n_{t}}
$$

$$
\begin{aligned}
& u_{z}= \mathrm{K}_{z} f+\mathrm{K} f_{z}+\int_{-\pi}^{\pi}\left(\mathrm{E}_{z} f+\mathrm{E} f_{z}\right) d t \\
&= \mathrm{K}_{z} f+\mathrm{K} f_{z}+\int_{-\pi}^{\pi}\{f[n(z, t)]\}\left[\mathrm{E}_{z}-\left(\mathrm{E} n_{z} / n_{t}\right)_{t}\right] d t+j, \\
& j=\left(f \mathrm{E} n_{z} / n_{t}\right)_{t=\pi}-\left(f \mathrm{E} n_{z} / n_{t}\right)_{t=-\pi} \\
& \quad+\sum_{\nu=1}^{p} f\left[n\left(z, a_{\nu}\right)\right] \lim _{\epsilon \rightarrow 0}\left[\left(\mathrm{E} n_{z} / n_{t}\right)_{t=a_{\nu}+\epsilon}-\left(\mathrm{E} n_{z} / n_{t}\right)_{t=a_{\nu}-\epsilon}\right],
\end{aligned}
$$


$t=a_{\nu} \equiv a_{\nu}(z), \nu=1,2, \cdots, p$, denoting the zeros of $n_{t}(z, t)$ in the interval $E[-\pi<t<\pi]$.

Differentiating (3.1) and (3.3) with respect to $\bar{z}$ and inserting the obtained expressions into (3.2) we have

$$
\mathrm{L}(u)=f \mathrm{~L}(\mathrm{~K})+\mathrm{K}_{\mathrm{z}} f_{z}+\int_{-\pi}^{\pi} f(n(z, t))\left[\mathrm{L}(\mathrm{E})-\left(\mathrm{E}_{z} n_{z} / n_{t}\right)_{t}\right] d t+j_{z}=0 .
$$

(3.4) must hold for any analytic function $f$ which is regular at the origin. The expression (3.4) is a sum of terms $M^{(k)} N^{(k)}$ and $\int_{-\pi}^{\pi} M^{(p)} N^{(p)} d t$, where $N^{(m)}, m=1,2, \cdots$, depend upon $\mathbf{E}, \mathrm{K}, n$ and (in the second expression) on $t$ but are independent of $f$, and $M^{(m)}$ depend also on $f$. If all $N^{(m)}$ vanish, that is, if

$$
\begin{aligned}
& N^{(1)} \equiv \mathrm{L}(\mathrm{E})-\left(\mathrm{E}_{\bar{z}} n_{z} / n_{t}\right)_{t}=0, \\
& N^{(2)} \equiv \mathrm{K}_{\bar{z}}=0, \\
& N^{(3)} \equiv \mathrm{L}(\mathrm{K})=0, \\
& N_{0}^{(4)} \equiv\left[\left(\mathrm{E} n_{z} / n_{t}\right)_{\bar{z}}\right]_{t=-\pi}=0, \\
& N_{\nu}^{(4)} \equiv\left(\mathrm{E}_{\bar{z}} n_{z} / n_{t}\right)_{t=a_{\nu}+\epsilon}-\left(\mathrm{E}_{\bar{z}} n_{z} / n_{t}\right)_{t=a_{\nu}-\epsilon}=0, \nu=1,2, \cdots, p, \\
& N_{p+1}^{(4)} \equiv\left[\left(\mathrm{E} n_{z} / n_{t}\right)_{\bar{z}}\right]_{t=\pi}=0,
\end{aligned}
$$

then clearly (3.1) will be a solution of (3.2). Therefore a sufficient condition in order that the operator (3.1) generate solutions of $L(u)=0$ is that $\mathbf{E}$ fulfills the differential equation $N^{(1)}=0$ and satisfies the additional conditions $\left({ }^{12}\right)$ $N_{v}^{(4)}=0, \nu=0,1, \cdots p, p+1$, and that $\mathrm{K}$ is a solution of (3.6) and (3.7).

However it could happen that two terms, for example $M^{(k)} N^{(k)}$ and $\int_{-\pi}^{\pi} M^{(s)} N^{(s)} d t$, can be combined into one term of a similar structure, say

$$
M^{(k)} N^{(k)}+\int_{-\pi}^{\pi} M^{(s)} N^{(s)} d t=\int_{-\pi}^{\pi} M N d t
$$

where $N$ is independent of $f$. This would mean that the number of conditions (3.8) is reduced. It is therefore of importance to show that certain relations of the form (3.9) can never occur.

\section{LEMma 3.1. A relation}

$$
\left\{f\left[n\left(z, a_{v}\right)\right] \mathrm{E}_{\tilde{z}} n_{z} / n_{t}\right\}+\int_{-\pi}^{\pi} f[n(z, t)] N^{(1)} d t=\int_{-\pi}^{\pi} f[n(z, t)] N d t,
$$

where $N$ is independent of $f$, is impossible unless $n(z, t)$ is independent of $t$.

Proof. (3.10) can be written in the form

(12) We denote the points $-\pi$ and $\pi$ by $a_{0}$ and $a_{p+1}$, respectively. 


$$
f\left[n\left(z, a_{v}\right)\right] \mathrm{E}_{\bar{z}} n_{z} / n_{t}=\int_{-\pi}^{\pi} f[n(z, t)] S(z, \bar{z}, t) d t
$$

where $S(z, \bar{z}, t)=N-N^{(1)}$ is a fixed function which is independent of $f$. But a relation (3.11) is impossible. Assume, on the contrary, that a relation (3.11) holds. Then for some value of $z$, say $z=z_{0}, n=n\left(z_{0}, t\right),-\pi \leqq t \leqq \pi$, will represent a curve in the complex plane, and, of course, $n\left(z_{0}, a_{\nu 0}\right), a_{\nu 0}=a_{\nu}\left(z_{0}\right)$, is only a point of it.

Assume at first that $\left[\mathrm{E}_{\bar{z}}\left(z_{0}, \bar{z}_{0}, a_{\nu 0}\right) n_{z}\left(z_{0}, a_{\nu 0}\right) / n_{t}\left(z_{0}, a_{\nu 0}\right)\right] \not \neq 0$. Then we can determine a function $f$ which is sufficiently large at the point $n\left(z_{0}, a_{v 0}\right)$ and sufficiently small on the remaining part of the curve $n=n\left(z_{0}, t\right),-\pi \leqq t \leqq \pi$, so that the right-hand side of (3.11) will be smaller than the left-hand side. Therefore such a relation is impossible.

If further we assume that $\left[\mathrm{E}_{z}\left(z_{0}, \bar{z}_{0}, a_{\nu 0}\right) n_{z}\left(z_{0}, a_{\nu 0}\right) / n_{t}\left(z_{0}, a_{\nu 0}\right)\right]=0$ then we may determine a function $f$ so that the real part of $f$ in the neighborhood of $n\left(z_{0}, \pi\right)$ is sufficiently large, and which is on the remaining part of the curve sufficiently small so that the right-hand side of (3.11) is not zero.

In a similar way one can show that it is impossible that a relation

$$
\begin{aligned}
f(z) \mathrm{L}(\mathrm{K})=f\left[n\left(z, a_{v}\right)\right] \mathbf{E}_{\bar{z}}\left(z, \bar{z}, a_{v}\right) n_{z}\left(z, a_{\nu}\right) / n_{t}\left(z, a_{v}\right), & \\
a_{\nu} & =0,1,2, \cdots, p, p+1,
\end{aligned}
$$

holds unless $n\left(z, a_{v}\right)=z$.

Finally it is evident that no relation

$$
f_{z}(z)=\int_{-\pi}^{\pi} N(z, \bar{z}, t) f[n(z, t)] d t \quad \text { or } \quad f_{z}(z)=f\left[n\left(z, a_{v}\right)\right]
$$

exists since we may determine a function $f$ which is smaller than 1 in absolute value on the integration curve $n=n(z, t),-\pi \leqq t \leqq \pi, z$ fixed, and possesses a derivative at the point $z$ which is larger than the above integral and $f\left[n\left(z, a_{v}\right)\right]$.

Summarizing our reasoning we obtain the following:

THEOREM 3.1. Let the functions $\mathbf{E}(z, \bar{z}, t)$ and $n(z, t)$ satisfy equation (3.5) and the additional conditions

$$
\begin{aligned}
N_{0}^{(4)} \equiv & \mathrm{E}_{\bar{z}}(z, \bar{z},-\pi) n_{z}(z,-\pi) / n_{t}(z,-\pi)=0, \\
N_{p+1}^{(4)} \equiv & \mathrm{E}_{\bar{z}}(z, \bar{z}, \pi) n_{z}(z, \pi) / n_{t}(z, \pi)=0, \\
N_{\nu}^{(4)} \equiv & \lim _{\rightarrow \rightarrow 0}\left\{\left[\mathrm{E}_{\bar{z}}(z, \bar{z}, t) n_{z}(z, t) / n_{t}(z, t)\right]_{t=a_{\nu}+\epsilon}\right. \\
& \left.-\left[\mathrm{E}_{\bar{z}}(z, \bar{z}, t) n_{z}(z, t) / n_{t}(z, t)\right]_{t=a_{\nu}-\epsilon}\right\}=0, \quad \nu=1,2, \cdots, p,
\end{aligned}
$$

where $t=a_{\nu} \equiv a_{\nu}(z),|z|<\rho, \rho>0$ sufficiently small, are the roots of $n_{t}(z, t)=0$ 
which lie on the interval $E[-\pi<t<\pi]$. Let $\mathrm{K}$ satisfy the equations (3.6) and (3.7).

Then the operator (3.1) transforms any analytic function of a complex variable which is regular at every point $n=n(z, t),-\pi \leqq t \leqq \pi,|z|<\rho, \rho>0$ suffciently small, into a solution of equation $\mathrm{L}(u)=0$. (See (3.2).)

If $n(z, \pi)=n(z,-\pi)$ then both first equations of (3.8) have to be replaced by

$$
\mathrm{E}_{\bar{z}}(z, \bar{z}, \pi) n_{z}(z, \pi) / n_{t}(z, \pi)=-\mathrm{E}_{z}(z, z,-\pi) n_{z}(z,-\pi) / n_{t}(z,-\pi) .
$$

If some functions $n\left(z, a_{\nu}(z)\right), \nu=0,1,2, \cdots, p+1$, say $n\left(z, a_{\nu, \mu}(z)\right)$, $\mu=1,2, \cdots, k$ (see footnote 12), are equal then the corresponding expressions $N_{\nu, \mu}^{(4)}$ can be combined in one equation

$$
\sum_{\mu=1}^{k} N_{\nu, \mu}^{(4)}=0 .
$$

If finally $n\left(z, a_{\nu, \mu}(z)\right)=z$ then $N^{(3)}=0$ (see (3.7)) can be combined with (3.14), and instead of (3.14) and (3.7) we have one equation

$$
\sum_{\mu=1}^{k} N_{\nu, \mu}^{(4)}+\mathrm{L}(\mathrm{K})=0 .
$$

In the case where $\mathfrak{i}^{1}$ is a closed curve the considerations are similar but slightly more complicated.

From now on $\mathrm{L}$ will again denote the expression introduced in (2.3) and $\mathbf{E}$ the corresponding generating function.

2. Since $u(z, \bar{z})=\sum_{m=0}^{\infty} \sum_{n=0}^{\infty} A_{m n} z^{m} \bar{z}^{n}$ are (complex) solutions of the equation $\mathrm{L}(u)=0$, they are determined if $\sum_{m=0}^{\infty} A_{m 0} z^{m}$ and $\sum_{m=0}^{\infty} A_{0 m} \bar{z}^{m}$ are given. If $u \in \mathcal{C}\left(\mathbf{E}, \mathbf{K}, n, \mathfrak{i}^{1}\right)$ then for many $\left(\mathbf{E}, \mathbf{K}, n, \mathfrak{i}^{1}\right), \sum_{m=0}^{\infty} A_{m 0} z^{m}$ can be an arbitrary analytic function of a complex variable, which is regular at origin. 'On the other hand if $f[n(0, t)]=c_{0}, c_{0}$ independent of $t$, then the $A_{0 m}$ are determined essentially by the operator, within $f(0)$ and $c_{0}$. Indeed substituting $z=0$ into

$$
\sum_{m=0}^{\infty} \sum_{n=0}^{\infty} A_{m n} z^{m \bar{z}^{n}}=\mathrm{K}(z, \bar{z}) f(z)+\int \mathrm{E}(z, \bar{z}, t) f[n(z, t)] d t
$$

we obtain

$$
\sum_{n=0}^{\infty} A_{0 n} \bar{z}^{n}=\mathrm{K}(0, \bar{z}) f(0)+c_{0} \int \mathrm{E}(0, \bar{z}, t) d t,
$$

where the integration is taken over $\mathfrak{i}^{1}$.

3. We proceed now to the determination of the matrix $\left\{\tau_{m n}\right\}$ and the function $\sum_{m=0}^{\infty} A_{0 m} \bar{z}^{m}$ which corresponds to a given operator of the form (3.1). For the sake of simplicity we assume that

$$
\mathfrak{i}^{1}=E[-\pi<t<\pi], \quad \mathrm{K}=0,
$$


and that $n(z, t)$ is an analytic function of $z$ which is regular at $z=0$ for $t \in \mathfrak{i}^{1}$. Hence we may write

$$
n(z, t)=n_{0}(t)+n_{1}(t) z+n_{2}(t) z^{2}+\cdots, \quad|z| \leqq \rho, t \in \mathfrak{i}^{1},
$$

where $\rho>0$, sufficiently small.

Let now $\mathbf{E}(z, \bar{z}, t),|z| \leqq \rho,|\bar{z}| \leqq \rho, t \in \mathfrak{i}^{1}$, be a solution of (3.5) which satisfies the initial value conditions (3.8), let

$$
\mathrm{E}(z, 0, t)=H_{0}(t)+H_{1}(t) z+H_{2}(t) z^{2}+\cdots, t \in \mathfrak{i}^{1}, \quad|z| \leqq \rho,
$$

and

$$
f(z)=\sum_{n=0}^{\infty} a_{n} z^{n}, \quad|z| \leqq \rho .
$$

Substituting these series developments into $\int_{-\pi}^{\pi} \mathrm{E}(z, 0, t) f[n(z, t)] d t$, we obtain

$$
U(z, 0)=\sum_{n=0}^{\infty} A_{n 0} z^{n}=\int_{-\pi}^{\pi} \mathrm{E}(z, 0, t) f[n(z, t)] d t=\sum_{n=0}^{\infty}\left(\sum_{m=0}^{\infty} \tau_{m n} a_{m}\right) z^{n} .
$$

A formal computation yields

$$
\begin{aligned}
\tau_{00} & =\int H_{0} d t, \quad \tau_{10}=\int H_{0} n_{0} d t, \\
\tau_{20} & =\int H_{0} n_{0}^{2} d t, \cdots, \\
\tau_{01} & =\int H_{1} d t, \quad \tau_{11}=\int\left(H_{1} n_{0}+H_{0} n_{1}\right) d t, \\
\tau_{21} & =\int\left(H_{1} n_{0}^{2}+2 H_{0} n_{0} n_{1}\right) d t, \cdots, \\
\tau_{02} & =\int H_{2} d t, \quad \tau_{12}=\int\left(H_{2} n_{0}+H_{1} n_{1}+H_{0} n_{2}\right) d t, \\
\tau_{22} & =\int\left[n_{0}^{2} H_{2}+2 H_{1} n_{0} n_{1}+H_{0}\left(2 n_{0} n_{2}+n_{1}^{2}\right)\right] d t, \cdots
\end{aligned}
$$

(the integration is to be taken over $\mathfrak{i}^{1}$ ) and

$$
\sum_{m=0}^{\infty} A_{0 m} \bar{z}^{m}=f(0) \int_{-\pi}^{\pi} \mathbf{E}(0, \bar{z}, t) d t .
$$

Thus using the procedure described above we may determine the matrix $\left\{\tau_{m n}\right\}$ and $\sum_{m=0}^{\infty} A_{0 m} \bar{z}^{m}$ for all possible integral operators of the form (3.1).

Another question is which among these operators are of interest. For some 
purposes one integral operator may be more valuable than another.

Remark. An interesting problem is the question how, for a given differential equation, to choose $\mathrm{K}, n$ and $\mathrm{i}^{1}$ in order that $\mathrm{E}$ is a function of $t$ which can be represented in a closed form in terms of elementary functions. See $[5, \S 3]$.

In many applications. it is necessary that $\mathbf{E}(z, \bar{z}, t)$ be an entire function of $z, \bar{z}$, or at least regular in some prescribed domain.

4. We obtain the operator introduced in [3] to which we referred in $\$ \S 1$ and 2 in the following way. Setting $\left({ }^{13}\right)$

$$
n(z, t)=(z / 2) \cos ^{2} t, \quad \mathrm{~K}(z, \bar{z})=0
$$

we obtain for $\mathbf{E}$ the equation (see (3.5))

$$
N^{(1)} \equiv\left[\left(z^{-1 / 2}\right) \cot t\left(\mathrm{E}_{\bar{z}}+a \mathrm{E}\right)\right]_{t}+\mathrm{L}(\mathrm{E})=0 .
$$

Among the solutions of (4.8) we choose the solution( $\left.{ }^{14}\right)$

$$
\mathrm{E}(z, \bar{z}, t)=\left[\exp \left(-\int_{0}^{\bar{z}} a d \bar{z}\right)\right]\left[1+\sum_{n=1}^{\infty}\left(\sin ^{2 n} t\right) \phi^{(n)}(z, \bar{z})\right],
$$

where $\phi^{(n)}(z, \bar{z})$ satisfy the following recurrence formulas:

$$
\begin{gathered}
\phi_{\bar{z}}{ }^{(1)}+2 z F=0, \\
(2 n+1) \phi_{\bar{z}}{ }^{(n+1)}+2 z\left(\phi_{\bar{z} \bar{z}}{ }^{(n)}+D \phi_{\bar{z}}{ }^{(n)}+F \phi^{(n)}-(n / z) \phi_{\bar{z}}{ }^{(n)}\right)=0, \\
n=1,2, \cdots, \\
\phi^{(n)}(z, 0)=0, n=0,1,2, \cdots, \text { where } D=\bar{a}-\int_{0}^{z} a_{z} d \bar{z}, F=-a_{z}-a \bar{a}+c .
\end{gathered}
$$

See $[3$, p. 1174].

Assuming that $A, B, C$ are entire functions of $z, \bar{z}$ it is possible to show that $\mathbf{E}$ is an entire function of $z, \bar{z}$. See [3, Theorem 2] $\left.{ }^{15}\right)$. As before mentioned, in the case under consideration the relations (2.10) and (2.11) are valid for the matrix $\left\{\tau_{m n}\right\}$ and for $\sum_{m=0}^{\infty} A_{0 m} \bar{z}^{m}$, respectively.

(13) We of ten use $t$ instead of $\sin t$, so that the operator under consideration assumes the form $\mathrm{P}(f)=\int_{-1}^{1} \mathrm{E}(z, \bar{z}, t) f\left(z\left(1-t^{2}\right) / 2\right) d t /\left(1-t^{2}\right)^{1 / 2}$. The factor $1 / 2$ in this operator causes some inconvenience. For instance, if the star domain $\xi^{2}$ is the regularity domain of $f$, then $u(z, \bar{z})$ is regular in 2(\$2). It would be more convenient to use $n(z, t)=z \cos ^{2} t$ rather than $(z / 2) \cos ^{2} t$. Unfortunately the second expression was introduced in previous papers, and we shall continue to use it in order to avoid confusion.

(14) The solution $\mathbf{E}$ of the form (3.17) has the advantage that $\mathbf{E}(z, 0, t)=1$, and therefore $u(z, 0)=\int_{-\pi}^{\pi} f\left((z / 2) \cos ^{2} t\right) d t$ so that $f(z)$ and $u(z, 0)$ are connected in this simple way.

It was suggested by the study of the equation $u_{z \bar{z}}+u / 4=0$. See [2] and footnote 11, p. 305 .

(15) This result follows if we choose, in Theorem 2 of $[3], \mathfrak{u}^{4}(0,0)=E[|z|<\infty,|\bar{z}|]<\infty$.

We note further that in (1.13), (1.16) and (1.20) of [3], " $(2 n-1)$ " has to be replaced by " $(2 n+1) "$ and in (1.23), (1.26), (1.27), (1.28), " $(2 n-3)$ " has to be replaced by " $(2 n-1) "$. Further in $(1.16)$, " $P_{\bar{z}}^{(2 n)}(z, \bar{z})$ " has to be replaced by " $P_{z}^{(2 n)}(z, \bar{z})$." 
The class of complex solutions of (1.3), $\mathcal{C}$, which has the generating function $\mathbf{E}$, introduced in (3.27), has many properties in common with the class of analytic functions of a complex variable $\left({ }^{16}\right)$. See $[4, \S \S 5-7]$ and $\S \S 5-9$ of the present paper. We denote it as the generating function of the first kind. The associate function of $u$ with respect to the above described operator is called the "R-associate" of $u(z, \bar{z}), \mathrm{R}(z \mid u)$. The relation

$$
\mathrm{R}(z \mid u)=\frac{2}{\pi} \int_{0}^{\pi / 2} z \sin \theta\left[d u\left(2 z \sin ^{2} \theta, 0\right) / d\left(z \sin ^{2} \theta\right)\right] d \theta+\pi^{-1} u(0,0)
$$

holds for R. In addition to $\mathrm{R}(z \mid u)$ often we consider the "Q-associate," for which we have simply

$$
Q(z \mid u)=u(z, 0)
$$

( $Q$ and $R$ are connected by the relation

$$
\left.\mathrm{R}(z \mid u)=[\Gamma(1 / 2)]^{-1}(2 z)^{1 / 2} d^{1 / 2} \mathrm{Q}(2 z \mid u) / d z^{1 / 2} .\right)
$$

For details see $[3, \S 1]$ and $[4, \S 3]$. A formal computation yields

$$
\begin{aligned}
& u(z, \bar{z})= {\left[\exp \left(-\int_{0}^{\bar{z}} a d \bar{z}\right)\right]\left[g(z)+\sum_{n=1}^{\infty} 2^{-2 n} \Gamma(2 n+1)[\Gamma(n+1)]^{-1}\right.} \\
&\left.\cdot Q^{(n)}(z, \bar{z}) \int_{0}^{z} \int_{0}^{z_{1}} \cdots \int_{0}^{z_{n-1}} g\left(z_{n}\right) d z_{n} \cdots d z_{1}\right] \\
&=\frac{2}{\pi} \int_{-\pi}^{\pi} \mathrm{E}(z, \bar{z}, \sin t) \\
& \cdot\left[\int_{0}^{\pi / 2} z \cos ^{2} \theta \frac{2 \sin d g\left(z \cos ^{2} t \sin ^{2} \theta\right)}{d\left(z \cos ^{2} t \sin ^{2} \theta\right)} d \theta+g(0)\right] d t .
\end{aligned}
$$

Remark. If $u=\sum A_{m n} z^{m} \bar{z}^{n} \in \mathcal{C}(\mathrm{E}), A_{00}$ real, where $\mathbf{E}$ is the generating function of the first kind and $\sum D_{m n} z^{m} \bar{z}^{n}$ its real part, then $\sum D_{m 0} z^{m}=\sum A_{m 0} z^{m}$ $+\exp \left[-\int_{0}^{z} \bar{a}(0, z) d z\right]$.

\section{Additional remarks.}

1. An alternate form for the representation of operators. For the purpose of applications it is of importance to obtain various forms of the operators previously described in this paper.

LEMMa 4.1. Let

$$
\left|\Psi^{(v)}(z, \bar{z})\right|<C|z / r| v, \bar{z} \text { conjugate to } z .
$$

\section{The operator}

(16) We note that of ten we write $\mathcal{C}(\mathbf{E})$ instead of $\mathcal{C}\left[\left(1-t^{2}\right)^{-1 / 2} \mathrm{E}(z, \bar{z}, t), 0, z\left(1-t^{2}\right) / 2\right.$, $E(-1<t<1)]$. 


$$
\mathrm{P}(f)=\int_{-1}^{1} \mathrm{E}_{1}(z, \bar{z}, t) f\left(z\left(1-t^{2}\right) / 2\right) d t /\left(1-t^{2}\right)^{1 / 2}
$$

where

$$
\mathrm{E}_{1}(z, \bar{z}, t)=\sum_{p=0}^{\infty} \Psi^{(v)}(z, \bar{z}) t^{2 v}
$$

can be written in the form

$$
\left.\mathrm{P}(f)=\sum_{\nu=0}^{\infty} \frac{z^{\nu} f^{[(\nu)]}(0) \Gamma(\nu+1 / 2)}{2^{\nu} \Gamma(\nu+1)} \mathrm{H}^{[\nu]}(1, z, \bar{z}), f^{[(\nu)]}(0)=\frac{d^{\nu} f(z)}{d z^{\nu}}\right]_{z=0},
$$

where

$$
\begin{aligned}
\mathrm{H}(\zeta, z, \bar{z}) & =\sum_{n=0}^{\infty} \zeta^{n} \Psi^{(n)}(z, \bar{z}) \Gamma(n+1 / 2) / \Gamma(n+1), \\
\mathrm{H}^{[p]}(\zeta, z, \bar{z}) & =\int_{0}^{\zeta} \int_{0}^{\zeta_{1}} \cdots \int_{0}^{\zeta_{n-1}} \mathrm{H}\left(\zeta_{n}, z, \bar{z}\right) d \zeta_{n} \cdots d \zeta_{2} d \zeta_{1},
\end{aligned}
$$

$|z|<\rho$, where $\rho=\min (r, R), R$ being the radius of convergence of $f(z / 2)$.

Proof. Substituting

$$
f\left(z\left(1-t^{2}\right) / 2\right)=\sum_{n=0}^{\infty} 2^{-n} z^{n} f^{[(n)]}(0)\left(1-t^{2}\right)^{n} / \Gamma(n+1)
$$

into (4.2) we obtain

$$
\int_{-1}^{1} \sum_{\nu=0}^{\infty} \Psi^{(v)}(z, \bar{z}) t^{2 \nu}\left[\sum_{n=0}^{\infty} 2^{-n} z^{n} f^{[(n)]}(0)\left(1-t^{2}\right) / \Gamma(n+1)\right] d t /\left(1-t^{2}\right)^{1 / 2} .
$$

Both series converge uniformly and absolutely in the circle $E[|z|<\rho]$. Interchanging the order of summation and integration and rearranging the terms we obtain

$$
\begin{aligned}
\sum_{\nu=0}^{\infty}\left[z^{p} f^{[(\nu)]}(0) \frac{\Gamma(\nu+1 / 2)}{\Gamma(\nu+1)}\left(\sum_{n=0}^{\infty} \Psi^{(n)}(z, \bar{z}) \frac{\Gamma(n+1 / 2)}{\Gamma(\nu+n+1)}\right)\right] \\
=\sum_{\nu=0}^{\infty} z^{\nu[(\nu)]}(0) \Gamma(\nu+1 / 2) \mathrm{H}^{[\nu]}(1, z, \bar{z}) / \Gamma(\nu+1) .
\end{aligned}
$$

LEMMA 4.2. If the coefficients $a, c$ of the equation (2.3) are entire functions of $z, \bar{z}$ and if $\mathbf{E}_{1}$ is the function $\mathbf{E}$ of the first kind which generates the solutions $u$ of (2.3), then representation (4.4) holds in the circle of convergence $E[|z| \leqq R]$ of $f(z / 2)$.

Proof. According to (1.27) of [3], for every $r$ there exists a constant $C$ 
such that

$$
\left|\Psi^{(\nu)}(z, \bar{z})\right|=\left|\Phi^{(v)}(z, \bar{z})\right| \leqq C|2 z / r|^{v}
$$

(see also (3.28)). Therefore $\mathrm{H}(\zeta, z, \bar{z})$ is an entire function of $z, \bar{z}$ for $|\zeta| \leqq 1$, and for every $r$ there exists a constant $C=C(r)$, such that

$$
\mathrm{H}^{[\nu]}(\zeta, z, \bar{z}) \leqq C / \Gamma(\nu+1),|z| \leqq r,|\bar{z}| \leqq r,|\zeta| \leqq 1 .
$$

Since $\lim \sup _{\nu \rightarrow \infty}\left[f^{(\nu)]}(0) / 2^{\nu} \Gamma(\nu+1)\right]^{1 / \nu}=1 / R,(4.4)$ converges for $|z|<R$. This implies Lemma 4.2.

$A n$ application of the operator (3.32). Let $u(z, \bar{z})=\sum A_{m n} z^{m} \bar{z}^{n}$ be a solution of (2.3), $u \in \mathcal{C}(\mathbf{E})$, where $\mathbf{E}$ is a generating function of the first kind. From the form (3.32) of the integral operator which transforms $u(z, 0)=\sum A_{m 0} z^{m}$ into $u(z, \bar{z})$, it follows that many relations of the sort indicated in II, p. 299, hold in the case of functions $u(z, \bar{z})$ of class $\mathcal{C}(\mathbf{E})$. Typical examples are:

1. Let $A_{m 0}=O\left(m^{-1-\epsilon}\right), \epsilon>0$. Then $g=u(z, 0)$ and all integrals in (3.32) are continuous on $|z|=1$, and since (3.32) converges uniformly there, $u(z, \bar{z})$ is also continuous there.

2. As is well known, it is possible to formulate, in terms of the $A_{m 0}$, $m=0,1,2, \cdots$, sufficient conditions in order that $\int_{0}^{z} u(z, 0) d z$ is continuous on $|z|=1$ and that $\operatorname{Re}[u(z, 0)]$ has a jump, as well as to determine the magnitude and location of this jump. $u(z, \bar{z})$ has at the same point on $|z|=1$ a similar singularity.

We note that the expressions for the location and the magnitude of the jump of $\operatorname{Re}\left\{[u(z, \bar{z})] / \exp \left(-\int_{0}^{\bar{z}} a(z, \bar{z}) d \bar{z}\right)\right\}$ do not involve the coefficients $a, c$ of equation (2.3). From the above results for complex solutions we may derive analogous results for (real) solutions $U(z, \bar{z})=\sum D_{m n} z^{m} \bar{z}^{n}=\operatorname{Re}(u)$. By the relation $2 U(z, 0)=u(z, 0)+\exp \left(-\int_{0}^{z} \bar{a}(0, z) d z\right) u(0,0)$ (see (2.9), (2.11)) we see that in addition to the $D_{m 0}, m=0,1,2, \cdots$, now only $a(z, 0)$ and $\bar{a}(0, z)$ will appear in the corresponding formulas.

In similar manner we can obtain various theorems on behaviour of solutions $u(z, \bar{z})$ and $U(z, \bar{z})$ in the neighborhood of singular points, results concerning methods of summation of $\sum A_{m n} z^{m} \bar{z}^{n}$, and so on.

2. Other types of integral operators. By a procedure similar to that developed in $\S 3$ we can determine the integral operators

$$
\begin{aligned}
\mathrm{P}(f)= & \mathrm{K}(z, \bar{z}) f(z)+\int \mathrm{E}^{(1)}(z, \bar{z}, t) f\left[n_{1}(z, t)\right] d t \\
& +\iint \mathrm{E}^{(2)}(z, \bar{z}, t, \tau) f\left[n_{2}(z, t, \tau)\right] d t d \tau,
\end{aligned}
$$

where the first integral is taken over $\mathfrak{i}^{1}$ and the double integral is taken over $\Im^{2}$, or those of more complicated structure, which transform analytic functions into solutions of (2.3). 
Another type of integral operator has the form

$$
\mathrm{P}(f)=f(z)+\int f[n(x, y, t)] \mathrm{E}(x, y, t) d t,
$$

where the integration is taken over $\mathfrak{i}^{1}$. This kind of operators will be discussed in another paper (in connection with differential equations of three variables).

Remark. In considering special types of differential equations, it is of interest to introduce also differential operators.

Generalizing the "method of cascades" we conclude that if the coefficients of the differential equation are connected by certain differential relations, then the totality of solutions can be represented in the form $\left({ }^{17}\right)$

$$
u(z, \bar{z})=\mathrm{D}(f) \equiv \sum_{k=0}^{n} B_{k}(z, \bar{z}) d^{k} f(z) / d z^{k} .
$$

Here $B_{k}$ are some fixed functions and $f(z)$ ranges over the totality of analytic functions. (See for details [5, footnote 5], and the literature indicated there.) The result formulated in Theorem 3.1 leads us to a great variety of integral operators, since for many functions $n(z, t)$ there exist functions $\mathbf{E}(z, \bar{z}, t)$ and $\mathrm{K}(z, \bar{z})$ satisfying the equations (3.5), (3.6), (3.7), and the conditions (3.8).

Further integral operators of considerable interest for applications can be obtained if we take for $\mathfrak{i}^{1}$ a closed curve $\left({ }^{18}\right)$ or if we introduce integral operators of different types, for example of the form (4.11).

We wish to add here two remarks concerning the linear operators.

Remark 1. In introducing operators (in a general manner) by matrices it is useful to make a certain classification of these operators.

A. Suppose that for every sequence $\left\{a_{m}\right\}$, for which $\sum_{m=0}^{\infty} a_{m}|r|^{m}<\infty, r>0$, the sums $\sum_{m=0}^{\infty} \tau_{m n} a_{m}$ converge and the corresponding function $p(z, \bar{z}) k(z, \bar{z})$ is regular in a sufficiently small circle $x^{2}+y^{2}<\rho^{2}, \rho>0$. If, simultaneously, the inverse operator transforms every function $p(z, \bar{z}) k(z, \bar{z})$ which is regular in a circle $x^{2}+y^{2}<\rho_{1}^{2}$ into a function $g(z)$ which is regular in a sufficiently small circle, say $x^{2}+y^{2}<r_{1}^{2}, r_{1}>0$, then the operator under consideration is said to belong to the type $A$.

$B$. If the operator is of type $A$ and in addition to this, proceeding in both directions, we always have $r=\rho$, then we say that the operator is of type B.

Remark 2. We concentrate in the present paper our attention on finding operators in the case of all equations (2.1) with analytic coefficients.

If, however, one limits himself to special classes of partial differential

${ }^{(17)}$ Clearly using the Cauchy formula we may express D as an integral operator of the type (3.1).

$\left({ }^{18}\right)$ By the procedure indicated in $[3, \S 5]$, every operator can be replaced by an integral operator $\int \mathrm{H}(z, \bar{z}, t) f(t) d t$, where the integration is taken over a closed integration curve $\mathfrak{i}^{1}$. We note that in this case $i^{1}$ can be any closed rectifiable Jordan curve which lies in the regularity domain of $f$ and includes the origin. 
equations, for instance to equations whose coefficients are connected by some relations, then for some special classes of equations there exist very interesting pairs of solutions $(U, W)$.

3. The conjugate function. As indicated in $\$ 3$ we shall make some remarks concerning the choice of the "conjugate" of a (real) solution $U$.

Many theorems on analytic functions can be formulated as statements about properties of a real harmonic function. There are, however, many results which essentially involve the real and the imaginary parts of an analytic function and which when formulated in terms of harmonic functions represent statements on pairs of harmonic functions (which are connected by the Cauchy-Riemann equations).

By introducing an operator which transforms an analytic function into a complex solution, $U+i V$, we associate with every $U$ a conjugate $V\left({ }^{19}\right)$.

There exist, however, other interesting possibilities of choosing the "conjugate."

For any of these choices of the "conjugate" $V$ we shall require (i) that the $V$ is uniquely (to within a fixed function multiplied by an arbitrary constant) determined by $U$; $\left(\mathrm{ii}\left({ }^{20}\right)\right)$ that the regularity domain of $V$ (in the real $x y$ plane) coincides with that of $\left.{ }^{21}\right) U(z, \bar{z}), \bar{z}$ conjugate to $z$; (iii) that $V$ has some basic properties which conjugates possess in the case of harmonic functions.

We obtain a choice which satisfies all these conditions, and which differs from that used before by requiring that $U$ and $V$ are connected by generalized Cauchy-Riemann equations:

$$
a_{11}^{(s)} U_{x}+a_{21}^{(s)} U_{y}+a_{31}^{(s)} U+a_{41}^{(s)}
$$

$$
\begin{array}{cl}
=a_{12}{ }^{(s)} V_{x}+a_{22}{ }^{(s)} V_{y}+a_{32}{ }^{(s)} V+a_{42}{ }^{(s)}, & s=1,2, \\
a_{k 1}{ }^{(1)} a_{k 2}{ }^{(2)}-a_{\kappa 1}{ }^{(2)} a_{\kappa 2}{ }^{(1)} \neq 0, & \kappa=1,2 .
\end{array}
$$

Clearly a generalized Cauchy's integral formula holds for such pairs of functions $\left({ }^{22}\right)$ (see $[4$, p. 145]), and therefore we are in the position to apply many

(19) In the case of harmonic functions it is very natural to require that both functions, $U$ and $V$, are connected by Cauchy-Riemann's equations. Any attempt to consider pairs of harmonic functions which are connected by other relations would probably lead to a highly complicated theory.

In contrast to this is the case of functions $U$ satisfying (2.3); there exist various combinations the study of which is of interest.

${ }^{(20)}$ We note that this property is not always satisfied by the conjugates obtained by means of an integral operator.

(21) The usual classification of singularities (poles, branch points, essential singularities) refers to analytic functions, that is, to the pairs of harmonic functions.

Therefore in particular we shall expect that the conjugate $V$ will be chosen in such a way that to each singularity of the associate corresponds a singularity of $U+i V$ at the same point, and that the classification of singularities of analytic functions can be used as a basis for a classification of those of $U+i V, \mathrm{~L}(U)=0$.

${ }^{22}$ ) The generalized Cauchy formula involves a "fundamental solution" $W(x, y ; \xi, \eta)$ $=A(x, y ; \xi, \eta) \log \left[(x-\xi)^{2}+(y-\eta)^{2}\right]+B(x, y ; \xi, \eta)$ of $(2.3)$. In this connection it is of importance 
of the methods of attack used in the theory of analytic functions of a complex variable, and we obtain analogous results.

4. An interpretation of the integral representation (1.2) with a generating function $\mathbf{E}$ of the first kind. The integral representation (1.2) where $\mathbf{E}$ is the generating function of the first kind permits an interpretation which sheds some light on the nature of our approach. In (2.3) let us replace $z$ by $X$ and $\bar{z}$ by $Y$ where $X$ and $Y$ are supposed to be real variables, and write $b$ instead of $\bar{a}$. The resulting equation

$$
u_{X Y}+a u_{X}+b u_{Y}+c u=0
$$

is of hyperbolic type. If now the values of $u(X, 0)=\sum_{n=0}^{\infty} A_{n 0} X^{n}$ and of $u(0, Y)=\sum_{n=0}^{\infty} A_{0 n} Y^{n}$ along intervals $E\left[0 \leqq X \leqq X_{0}\right]$ and $E\left[0 \leqq Y \leqq Y_{0}\right]$ of the $X$ and $Y$ axes, respectively, are given by a known formula

$$
u(X, Y)=u(0,0) R(X, Y, 0,0)+\int_{0}^{X} R(X, Y ; \xi, 0) h_{1}(\xi) d \xi
$$

$$
+\int_{0}^{Y} R(X, Y ; 0, \eta) h_{2}(\eta) d \eta
$$

$$
h_{1}(\xi)=\partial u(\xi, 0) / \partial \xi+b u(\xi, 0), \quad h_{2}(\eta)=\partial u(0, \eta) / \partial \eta+a u(0, \eta),
$$

$u(X, Y)=\sum_{m=0}^{\infty} \sum_{n=0}^{\infty} A_{m n} X^{m} Y^{n}$ is determined in the rectangle $E\left[0 \leqq X \leqq X_{0}\right.$, $\left.0 \leqq Y \leqq Y_{0}\right]$. Here $R(X, Y ; \xi, \eta)$ is the Riemann function. Since we assume that $a, b$ and $c$ as well as $\sum_{n=0}^{\infty} A_{n 0} X^{n}$ and $\sum_{n=0}^{\infty} A_{0 n} Y^{n}$ are analytic functions of real variables, the function $u=\sum_{m=0}^{\infty} \sum_{n=0}^{\infty} A_{m n} X^{n} Y^{n}$ is also an analytic function of two real variables, and can be analytically continued outside of the rectangle $E\left[0 \leqq X \leqq X_{0}, 0 \leqq Y \leqq Y_{0}\right]$.

$$
\begin{aligned}
u(X, Y)=\int_{-1}^{l}\left[\mathrm{E}_{1}(X, Y, t) f_{1}\left(\frac{X}{2}\left(1-t^{2}\right)\right)\right. & \\
& \left.+\mathrm{E}_{2}(X, Y, t) f_{2}\left(\frac{Y}{2}\left(1-t^{2}\right)\right)\right] d t /\left(1-t^{2}\right)^{1 / 2}
\end{aligned}
$$

$$
\begin{aligned}
& f_{1}\left(\frac{X}{2}\right)=\frac{2}{\pi} \int_{0}^{\pi / 2} X \sin \theta \frac{\partial u\left(X \sin ^{2} \theta, 0\right)}{\partial\left(X \sin ^{2} \theta\right)} d \theta+\frac{1}{\pi} u(0,0), \\
& f_{2}\left(\frac{Y}{2}\right)=\frac{2}{\pi} \int_{0}^{\pi / 2} Y \sin \theta \frac{\partial u\left(0, Y \sin ^{2} \theta\right)}{\partial\left(Y \sin ^{2} \theta\right)} d \theta,
\end{aligned}
$$

where $\mathbf{E}_{1}$ and $\mathbf{E}_{2}$ are the corresponding generating functions, gives a representation of $u$ in the whole regularity domain of $u$. (See Duke Math. J. vol. 6 (1940) p. 540.)

to study the "fundamental solutions" of (2.3) and to determine those among them which are especially suitable for certain purposes.

In particular, taking as basis the generalized Cauchy formula one can develop the theory of entire functions $U+i V$. 
Let us assume (in the remaining part of the section) that $z$ and $\bar{z}$ are not necessarily conjugate to each other. This means that we analytically continue $u$ to the complex values of $x$ and $y, z=x+i y, \zeta=x-i y$, and consider $u(z, \zeta)$ in the (four-dimensional) space of two complex variables $z$ and $\zeta$.

The analogue of intervals $E\left[0 \leqq X \leqq X_{0}, Y=0\right], E\left[0 \leqq Y \leqq Y_{0}, X=0\right]$ and the rectangle $E\left[0 \leqq X \leqq X_{0}, 0 \leqq Y \leqq Y_{0}\right]$ become the circles $E\left[|z| \leqq \mathrm{P}_{1}, \zeta=0\right]$, $E\left[|\zeta| \leqq \mathrm{P}_{2}, z=0\right]$ and the bicylinder $E\left[|z| \leqq \mathrm{P}_{1},|\zeta| \leqq \mathrm{P}_{2}\right]$, respectively. The integral (1.2) gives the representation of $u(z, \zeta)$ in terms of its values in the $z$ and in the $\zeta$ planes.

Since, however, we are limiting our considerations to the real domain (that is, to the real values of $x, y$ ), we must consider the function $u(z, \zeta)$ in the plane $\zeta=\underline{z}$. This last fact of ten complicates the formulation of the results.

Remark. Introducing as the argument of $f$ conveniently chosen expressions, for example, $x+i(1-y)^{1 / 2}$, we of ten obtain solutions of differential equations which are elliptic in one part of the $x y$-plane and hyperbolic in another.

\section{Singularities of functions of a class $\mathcal{C}(\mathbf{E})$}

5. Singularities corresponding to the poles and branch points of the associate function, in the case of a generating function of the first kind. The introduction of complex solutions $u$ of $\mathrm{L}(u)=0$ suggests the study of the singularities of the function $u=U+i V$, belonging to a class $\mathcal{C}(\mathbf{E})$, whose generating function is of the first kind.

If the associate function $f$ has a singularity at the point $z=2 \alpha$ and is regular in $E[|z| \leqq b, z \neq 2 \alpha], b>2|\alpha|>0$, then the function $u(z, \bar{z})$ obtained by applying (3.1) has a singularity at the point $z=\alpha$. (See [4, p. 150].)

Further if the $\mathrm{R}$ or $Q$ associate has at the points $z=2 \alpha$ and $z=\alpha$, respectively, a singularity of a certain type, for example a pole or a branch point, then as we shall show it is possible to describe the character and various properties of the singularity of $u(z, \bar{z})$ at the point $z=\alpha$.

Let the associate $Q(\zeta \mid u)$ possess a pole at the point $\zeta=\alpha$. Clearly, it will be sufficient for our purposes to consider

$$
\mathrm{Q}(\zeta \mid u)=(\alpha-\zeta)^{-1}
$$

Employing (3.32) we have

$$
\begin{aligned}
u(z, \bar{z})= & \exp \left(-\int_{0}^{\bar{z}} a d \bar{z}\right)\left\{\frac{1}{\alpha-z}+\frac{Q^{(1)}(z, \bar{z})}{2} \log \left(1-\frac{z}{\alpha}\right)+\cdots\right. \\
+ & \frac{\Gamma(2 n+1)}{2^{2 n} \Gamma(n+1)} Q^{(n)}(z, \bar{z})\left[\frac{(\alpha-z)^{n}}{\Gamma(n+1)} \log \left(1-\frac{z}{\alpha}\right)\right. \\
& \left.\quad+\sum_{k=1}^{n}(-1)^{k+1} a_{n, k} \alpha^{n-k} z^{k}\right]+\cdots
\end{aligned}
$$

where $a_{n, 1}=1 / \Gamma(n+1), a_{n, k}=[n \Gamma(n+1)]^{-1} C_{n, k}+k^{-1} a_{n-1, k-1}$. 
The associate $R(\zeta \mid u)$ can be written in a closed form; substituting the obtained expression into (3.33) we have

$$
\begin{aligned}
u(z, \bar{z})=\int_{-1}^{1} \mathrm{E}(z, \bar{z}, t) & {\left[\frac{z^{2}\left(1-t^{2}\right)^{2}}{\alpha\left[2 \alpha-z\left(1-t^{2}\right)\right]}+\frac{1}{4}\left(1-\frac{2 \alpha}{z\left(1-t^{2}\right)}\right)^{-3 / 2}\right.} \\
& \left.+\log \frac{\left(z\left(1-t^{2}\right)-2 \alpha\right)^{1 / 2}+\left(z\left(1-t^{2}\right)\right)^{1 / 2}}{\left(z\left(1-t^{2}\right)-2 \alpha\right)^{1 / 2}-\left(z\left(1-t^{2}\right)\right)^{1 / 2}}\right] \frac{d t}{\left(1-t^{2}\right)^{1 / 2}} .
\end{aligned}
$$

By (5.3) and [4, p. 150], $u$ is determined in the whole space except $z=2 \alpha$, where it possesses a combined pole and branch point of an infinite order.

The Riemannian surface of (5.3) consists of infinitely many sheets, connected at the branch points, $z=\alpha$ and $z=\infty$. Cutting the Riemannian surface of (5.3) along $E[z=\alpha s, 1 \leqq s \leqq \infty]$ we denote as the principal sheet of $u$ that sheet in which $\operatorname{Im}[\log (1-z / \alpha)]$ varies between 0 and $2 \pi$. Clearly if we let $z$ approach $\alpha$ along a path situated in the principle sheet we have

$$
\lim _{z \rightarrow \alpha}(\alpha-z) u(z, \bar{z})=\exp \left[-\int_{0}^{\bar{z}} a(z, \bar{z}) d \bar{z}\right] .
$$

We proceed in similar way if the $\mathrm{R}$-associate has a pole. Let

$$
\mathrm{R}(\zeta \mid u)=(\alpha-\zeta)^{-1} \text {. }
$$

Then

$$
Q(\zeta \mid u)=\frac{1}{2} \int_{-1}^{1} \frac{d t}{\left[2 \alpha-\zeta\left(1-t^{2}\right)\right]\left[1-t^{2}\right]^{1 / 2}}=\frac{\pi}{2[\alpha(\alpha-\zeta)]^{1 / 2}} .
$$

Thus we obtain

$$
\begin{aligned}
u(z, \bar{z})= & \int_{-1}^{1} \mathrm{E}(z, \bar{z}, t) \frac{d t}{\left[\alpha-z\left(1-t^{2}\right) / 2\right]\left(1-t^{2}\right)^{1 / 2}} \\
= & {\left[\exp \left(-\int_{0}^{\bar{z}} a(z, \bar{z}) d \bar{z}\right)\right]\left\{\frac{\pi}{[\alpha(\alpha-z)]^{1 / 2}}\right.} \\
& +\frac{Q^{(1)}(z, \bar{z})}{\alpha^{1 / 2}}\left[(\alpha-z)^{1 / 2}-\alpha^{1 / 2}\right] \\
& +\frac{\Gamma(5) Q^{(2)}(z, \bar{z})}{2^{4} \Gamma(3) \alpha^{1 / 2}}\left[-\frac{2}{3}(\alpha-z)^{3 / 2}+\frac{2}{3} \alpha^{3 / 2}-\alpha^{1 / 2} z\right] \\
& +\frac{\Gamma(7) Q^{(3)}(z, \bar{z})}{2^{6} \Gamma(4) \alpha^{1 / 2}}\left[\frac{4}{15}(\alpha-z)^{5 / 4}-\frac{4}{15} \alpha^{5 / 2}+\frac{2}{3} \alpha^{3 / 2} z-\frac{1}{2} \alpha^{1 / 2} z^{2}\right] \\
& +\cdots\},
\end{aligned}
$$


from which we may draw conclusions analogous to those obtained in the former case.

Since

$$
\begin{gathered}
\int_{0}^{z}\left(\alpha-z_{1}\right)^{n} d z_{1}=\frac{1}{n+1}\left[(\alpha-z)^{n+1}-\alpha^{n+1}\right] \\
\int_{0}^{z} \int_{0}^{z_{1}}\left(\alpha-z_{2}\right)^{n} d z_{2} d z_{1} \\
=\left[\frac{1}{(n+1)(n+2)}(\alpha-z)^{n+2}-\frac{\alpha^{n+2}}{(n+1)(n+2)}-\frac{\alpha^{n+1}}{n+1} z\right]
\end{gathered}
$$

we obtain from (5.2) the following theorem $\left({ }^{23}\right)$.

THEOREM 5.1. If the Q-associate $g(z)$ has a branch point of the nth order ( $n$ not an integer) then the corresponding function $u(z, \bar{z})$ has also a branch point of the nth order.

If $g(z)$ has a pole of the nth order at $\alpha$, and $|\alpha|<\infty$, then the corresponding function $u$ has a combined pole and a logarithmic branch point at $\alpha$.

If $g(z)$ has a pole at infinity, $u(z, \bar{z})$ has also a pole of the same order at infinity.

Definition. The above described singular point of $u(z, \bar{z})$ which corresponds to a pole of $n$th order of the $Q$-associate (that is, a combined pole and branch point if $|\alpha|<\infty$ and a pole if $\alpha=\infty$ ) will be denoted subsequently as a q-singular point of $n$th order.

Many theorems concerning connections between the coefficients of the series development of a function and the character of its singularities hold for functions $u \in \mathcal{C}(\mathbf{E}), \mathbf{E}$ being generating function of the first kind, for example:

LEMma 5.1. Let

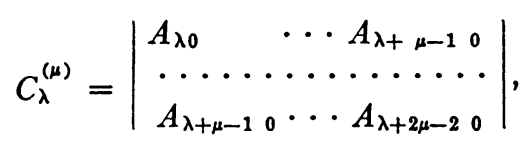

where $A_{00}+\sum_{m=1}^{\infty} \sum_{n=0}^{\infty} A_{m n} z^{m} \bar{z}^{n}$ is the series development of a function $u$, $u \in \mathcal{C}(\mathbf{E})$.

The necessary and sufficient conditions that all singularities of $u$ are q-singular points is that for suitable $M$ and $N$

$$
C_{\lambda}^{(\mu)}=0, \quad \lambda=M+1-N, M+2-N, \cdots ; \mu=N+1 .
$$

(23) We omit the formulation of the corresponding results for the case where the R-associate has a pole or a branch point. 
LEMMA 5.2. Let $u=A_{00}+\sum_{m=1}^{\infty} \sum_{n=0}^{\infty} A_{m n} z^{m} \bar{z}^{n}$ and $v=B_{00}+\sum_{m=1}^{\infty} \sum_{n=0}^{\infty} B_{m n} z^{m} \bar{z}^{n}$ be functions of the class $\mathcal{C}(\mathrm{E})$. If the determinants $C_{\lambda}^{(\mu)}$ corresponding to the $A_{n 0}$ satisfy the relations (5.10) and if

$$
A_{00} B_{00}=1, \quad \sum_{\nu=0}^{n} B_{n-\nu 0} A_{\nu 0}=0, \quad n=1,2,3, \cdots,
$$

then $v$ has only q-singularities, and the number of q-singular points (each singularity counted with the corresponding order of multiplicity) of $v$ equals the number of zeros of $\sum A_{m 0} z^{m}$.

6. The value distribution of functions of the class $\mathcal{C}(E)$. The analogy with the theory of analytic functions suggests the study of the value distribution of the functions belonging to the class $\mathcal{C}(\mathbf{E})$; that is, the investigation of how of ten a function $u(z, \bar{z}), \bar{z}$ conjugate to $z$, assumes a fixed (complex) value $b$.

Just as in the theory of entire functions of a complex variable, we shall investigate some relations which exist between the behaviour of the following three sets of numbers $\left({ }^{24}\right)$ which we can associate with a given function $u(z, \bar{z})$ of a class $\mathcal{C}(\mathbf{E})$ :

I. The coefficients, $A_{m n}$, of the series development of $\sum_{m=0}^{\infty} \sum_{n=0}^{\infty} A_{m n} z^{m} \bar{z}^{n}$ of $u(z, \bar{z})$, or some subsequences of the $A_{m n}$, say of $\left\{A_{m k}\right\}, k$ fixed, $m=0,1$, $2, \cdots$.

II. The $\max _{0 \leqq \phi \leqq 2 \pi}\left|u\left(r e^{i \phi}, r e^{-i \phi}\right)\right|$ when $r \rightarrow \infty$.

III. The points $z_{\nu}=z_{\nu}(b),\left|z_{\nu}\right| \leqq\left|z_{\nu+1}\right|$, where $u(z, \bar{z})$ assumes some fixed value, say $b$.

Remark. As was indicated in $\S 5$ a branch point of $u(z, \bar{z})$ of a certain type corresponds to a pole of the associate of the first or of the second type. A similar situation holds for various other types of the generating function, $\mathbf{E}$. This enables us to investigate functions $u(z, \bar{z}) \in \mathcal{C}(\mathbf{E})$ which possess denumerably many singular points of the above mentioned character, that is, functions whose associate with respect to some generating function $\mathbf{E}$ is meromorphic.

A simple example shows that, in general, we meet in the theory of functions of the class $\mathcal{C}(E)$ a situation which is quite different from that in the case of analytic functions. The functions $u_{n}(r, \phi)=\pi^{1 / 2} \Gamma(n+1 / 2) J_{n}(r) e^{i n \phi}$ $=2^{-n} \int_{-1}^{1} e^{i t r} r^{n} e^{i n \phi}\left(1-t^{2}\right)^{n-1 / 2} d t \in \mathcal{C}[\exp (i t r)], r^{2}=x^{2}+y^{2}$, are solutions of the equation $\Delta u+u=0$. Here $J_{n}$ are the Bessel functions of the $n$th order. If $r=r^{(0)}$ where $r^{(0)}$ is a zero of $J_{n}(r)$, then $u$ vanishes on the circle $|z|=r^{(0)}$, a situation which never can occur in the case of analytic functions.

However, if we generalize in a suitable manner certain notions from the theory of analytic functions the generalization of classical theorems on value distributions is possible.

${ }^{24}$ ) Clearly the behaviour of all three kinds of quantities depends upon the choice of the origin. The study of the dependence of the coefficients, $A_{m n}$, on the choice of the origin does not represent in many instances any essential difficulty. 
Let

$$
Z=X+i Y=u(z, \bar{z})=U(x, y)+i V(x, y)
$$

map the neighborhood of the point $z=z^{(0)}$ into the neighborhood of the point $Z=Z^{(0)}$. With every point $z^{(0)}$ we may associate the index $C\left(z^{(0)}\right)$ of the mapping.

Remark. In the following we shall assume for the sake of simplicity that the function $u(z, \bar{z})$ under consideration possesses only isolated $b$-points.

Let $\Omega^{2}$ be a simply connected domain in the $z$ plane and let $z_{\nu}$ be the points of $\Omega^{2}$ which are mapped into the points $Z=b$. As is well known

$$
\sum C\left[z_{\nu}(b)\right]=(2 \pi i)^{-1} \int d[\log u(z, \bar{z})-b]
$$

where the sum is taken over the $z_{\nu}(b) \in \Omega^{2}$, and the integration is taken over $\mathfrak{l}^{1}$, the boundary curve of $\Omega^{2}$.

If, in particular, $\Omega^{2}$ is the circle of radius $a$ with the center at the origin, we shall write

$$
\sum C\left[z_{\nu}(b)\right]=n\left[a,(u(z, \bar{z})-b)^{-1}\right],
$$

where the sum is taken over the $z_{\nu}(b) \in \Omega^{2}$.

There exists an important special case of a generating function $\mathbf{E}$, when it is possible to develop for the entire functions of the class $C(\mathbf{E})$ a theory similar to that of the entire analytic functions of a complex variable.

Suppose that $\mathrm{E}(z, \bar{z}, t)$ has the property that introducing the variables $z$ and $s=(z \bar{z})^{1 / 2}$ instead of $z, \bar{z}$ we have

$$
\mathbf{E}(z, \bar{z}, t)=\mathbf{E}^{(1)}(z, s, t) \equiv \mathbf{E}\left(z, s^{2} / z, t\right),
$$

where $\mathbf{E}^{(1)}$ is an entire function of two complex variables, $z$ and $s$, for $|t| \leqq 1$.

Let us indicate an example of a function $\mathbf{E}$ possessing the above property. If we assume that $u$ satisfies the equation $\Delta u+F(z, \bar{z}) u=0$, $F=z\left(\sum_{p=1}^{P} \sum_{m=1}^{M} A_{m p}^{(1)} z^{m} \bar{z}^{p}\right)$, where $A_{m p}^{(1)}=0$ for $m<p$, then the generating function of the first kind will be of the required type. For, if we write $\mathrm{E}=1+\sum_{n=1}^{\infty} t^{2 n} \Phi^{(2 n)}$ and $P^{(2 n)}=z^{-n} \Phi_{\bar{z}}^{(2 n)}$, we obtain (see $[3$, p. 1174]):

$$
P^{(2)}=-2 F, \quad(2 n+1) P^{(2 n+2)}=-2 P_{z}^{(2 n)}+F \int_{0}^{\bar{z}} P^{(2 n)} d \bar{z} .
$$

Let $A_{m p}^{(k)}$ denote a sequence of numbers which possesses the property that $A_{m p}^{(\boldsymbol{k})}=0$ for $m<p$. Then $\Phi^{(2)}=-2 z^{2} \int_{0}^{\bar{z}} A_{m p}^{(1)} z^{m} \bar{z}^{p} d \bar{z}=\sum A_{m p}^{(2)} z^{m} \bar{z}^{p}$. Assume now that $\phi^{(2 n)}=\sum A_{m p}^{(n)} z^{m} \bar{z}^{p}$. Then

$$
P^{(2 n)}=z^{-n} \bar{z}^{-1} \sum A_{m p}^{(8)} z^{m} \bar{z}^{p}
$$

and 


$$
\begin{aligned}
P^{(2 n+2)} & =z^{-n} \bar{z}^{-1}\left[z^{-1} \sum A_{m p}^{(n)} z^{m} \bar{z}^{p}+z\left(A_{m p}^{(1)} z^{m} \bar{z}^{p}\right) \int_{0}^{\bar{z}} \sum A_{m p}^{(n)} z^{m} \bar{z}^{p} d \bar{z}\right] \\
& =z^{-(n+1)} \bar{z}^{-1}\left[A_{m p}^{(n) z^{m} \bar{z}^{p}}+z^{2} \bar{z}^{-1} \sum A_{m p}^{(n)} z^{m} \bar{z}^{p}\right] .
\end{aligned}
$$

(Note that the $A_{m p}^{(n)}$ do represent different numbers in the above expressions.) Therefore

$$
\Phi^{(2 n+2)}=\sum A_{m p}^{(n+1) z^{m} \bar{z}^{p}} .
$$

For the sake of brevity, in the following the classes $C(\mathbf{E})$ whose generating function $\mathbf{E}$ possesses the above property will be denoted by $C \dagger(\mathbf{E})$.

The basic idea of the applied method consists in considering simultaneously with $u(z, \bar{z})$ the family of functions

$$
v_{s}(z)=\int_{-1}^{1} \mathbf{E}^{(1)}(z, s, t) f\left(z\left(1-t^{2}\right) / 2\right) d t /\left(1-t^{2}\right)^{1 / 2}, \quad 0<s<\infty,
$$

where $f$ is the associate of the first kind of $u \cdot v_{s}(z)=\sum c_{n}(s) z^{n}$ is a function of a real variable $s$ and of the complex variable $z$. In the following we extend the domain of variation of $s$ to the whole complex plane. $v_{s}(z)=\sum c_{m n} s^{m} z^{n}$ becomes then an analytic function of two complex variables, $s$ and $z$. Clearly, it is rather simple to express the $c_{m n}$ in terms of the $A_{m n}$, or some subsequences of the $A_{m n}$. Using the classical method we can then determine an upper bound $\mu(Z, S)$ for the growth of $\sum\left|c_{m n}\right||s|^{m}|z|^{n}$ on the hypersurface $E[|z|=Z$, $|s|=S, 0<S<\infty, Z=K S], K$ constant. Clearly

$$
\max _{|z|=K S}\left|v_{s}(z)\right| \leqq e^{\mu(s, K S)},
$$

$s>0$.

Applying now the classical theorems of the theory of entire functions of a complex variable, we obtain upper bounds for the quantities

$$
\begin{gathered}
m\left[s,\left(v_{s}(z)-b\right)^{-1}\right], \\
m\left[r,\left(v_{s}(z)-b\right)^{-1}\right]=\frac{1}{2 \pi i} \int_{0}^{2 \pi}\left[\log ^{+}\left|\frac{1}{v_{s}\left(r e^{i \phi}\right)-b}\right| d \phi\right]
\end{gathered}
$$

and

$$
n\left[s,\left(v_{s}(z)-b\right)^{-1}\right],
$$

where (6.8) denotes the number of the $b$ points of $v_{s}(z)$ in $|z| \leqq s$.

On the other hand, as we shall show in $\S 8$, the quantities connected with growth and density of $b$ points of $u(z, \bar{z}), \bar{z}$ conjugate to $z$, can be expressed in a simple way by (6.7) and (6.8).

We proceed now to a more detailed exposition of this method of attack.

7. Some auxiliary theorems of the theory of entire functions of two complex variables. 
THEOREM 7.1(25). Let

$$
V(s, z)=\sum_{m=0}^{\infty} \sum_{n=0}^{\infty} C_{m n} s^{m} z^{n}, \quad C_{m n} \geqq 0,
$$

be an entire function of two complex variables, with non-negative coefficients, and let

$$
\mu(S, Z)=\max _{|\delta|-S,|z|-Z}|V(s, z)|
$$

and

$$
\rho=\rho(K)=\limsup _{t \rightarrow \infty} \frac{\log \log \mu(t, K t)}{\log t}, \quad 0<\rho<\infty,
$$

be the order of growth of $\mu$ on the hypersurface

$$
E[|s|=t,|z|=K t, 0<t<\infty] \text {. }
$$

Then

$$
\rho=\rho_{1}, \quad \rho_{1} \equiv \limsup _{n+m \rightarrow \infty} \frac{(n+m) \log (n+m)}{\log \left(1 / C_{m n}\right)+n \log (1 / K)} .
$$

Proof. A. We shall prove at first that $\rho$ is not smaller than the right-hand side of (7.4). By Cauchy's formula we have

$$
C_{m n} \leqq \frac{\mu(|s|,|z|)}{|s|^{m}|z|^{n}} \text { or } C_{m n} K^{n}|s|^{n+m} \leqq \mu(|s|, K|s|) .
$$

Let us assume that $k$ is chosen in such a way that for $|s| \geqq S_{0}, S_{0}$ sufficiently large,

(7.6) $\mu(|s|, K|s|) \leqq \exp \left(|s|^{k}\right)$, and therefore $C_{m n} K^{n} \leqq \exp \left(|s|^{k}\right) /|s|^{m+n}$. Substituting $|s|=[(m+n) / k]^{1 / k}$ we obtain

$$
C_{m n}^{1 /(n+m)} K^{n /(n+m)} \leqq\left(\frac{e k}{m+n}\right)^{1 / k}
$$

that is,

$$
\left(\frac{1}{n+m}\right)\left[\log \frac{1}{C_{m n}}+n \log \frac{1}{K}\right] \geqq \frac{\log (m+n)-\log k-1}{k}
$$

(25) Similar results were obtained by Baumgarten [1] and Sire [8, p. 11]. Since, however, we need inequalities which are different from those obtained by Sire and Baumgarten, we derive here directly the relation (7.4). The procedure is clearly a trivial generalization of analogous considerations in the case of one complex variable. We note that for the sake of brevity we omit considerations which lead to sharper results. 
or

$$
k \geqq \frac{(n+m) \log (m+n)}{\log \left(1 / C_{m n}\right)+n \log (1 / K)} \quad(m+n \text { sufficiently large })
$$

since for sufficiently large $(m+n)$ we can neglect $\log k+1$. Thus

$$
\rho \geqq \limsup _{n+m \rightarrow \infty} \frac{(n+m) \log (n+m)}{\log \left(1 / C_{m n}\right)+n \log (1 / K)} .
$$

Thus the order, $\rho$, of growth is not smaller than the right-hand side of (7.4).

B. We shall now prove that $\rho$ is not larger than the right-hand side of (7.4). Let $\rho_{1}<\kappa$. We write

$$
N(s)=2^{*}|s|{ }^{*}(e \kappa) .
$$

Then

$$
\left(\frac{e \kappa}{m+n}\right)^{1 / \kappa}|s| \leqq 1 / 2 \text { for } m+n \geqq N(s) \text { : }
$$

From $\rho_{1}<\kappa$ we can work backwards from (7.8) to get (7.7), $k$ being replaced by $\kappa$ in both expressions and $m+n$ sufficiently large. Therefore

$$
\begin{aligned}
\mu(|s|, K|s|) & \leqq C_{00}+C_{10}|s|+C_{01}|z|+\cdots \\
& \leqq \sum_{m+n \leqq N(s)} C_{m n}|s|^{m}|z|^{n}+\left(1 / 2^{N(s)}\right)[N(s)+3] .
\end{aligned}
$$

For sufficiently large $|s|, N(s)$ becomes arbitrarily large, and therefore

$$
\mu(|s|, K|s|) \leqq[N(s)+2]^{2} C(s) / 2, \quad C(s)=\max \left(C_{m n} K^{n}|s|^{m+n}\right) .
$$

Using (7.7) (with $\kappa$ instead of $k$ ) we obtain by a formal computation

(7.14) $C_{m n} K^{n}|s|^{m+n}=C_{m n}|s|^{m}|z|^{n} \leqq\left(\frac{e \kappa}{m+n}\right)^{(m+n) / \kappa}|s|^{m+n}$, slarge enough.

The maximum of the right-hand expression (when $(m+n)$ varies) is assumed for $m+n=\kappa|s|$. Substituting this value in the right-hand side of (7.14) we obtain

$$
C(s) \leqq\left(\frac{e}{|s|^{\kappa}}\right)^{(m+n) / \kappa}|s|^{m+n}=\exp (|s| \kappa) .
$$

By (7.13), (7.10) and (7.15) we have

$$
\mu(|s|, K|s|) \leqq 2^{2 \kappa}|s|^{2{ }^{2}} e^{2} \kappa^{2} \exp \left(|s|{ }^{*}\right),
$$

which yields the assertion $\mathrm{B}$, and thus completes the proof of the Theorem 7.1. 
Remark. $\rho=\rho(K), 0<K_{0} \leqq K \leqq K_{1}<\infty, \rho<\infty$, the order of growth of $\mu(|s|, K|s|)$ is independent of $K$.

This follows immediately from (7.4): divide the numerator and the denominator by $n$. Since $(1+(m / n)) \log (n+m) \rightarrow \infty$ for $(m+n) \rightarrow \infty$, $n^{-1} \log \left(1 / C_{m n}\right) \rightarrow \infty$ for $(m+n) \rightarrow \infty$, and therefore $\log (1 / K)$ can be neglected.

If the series development

$$
u(z, \bar{z})=\sum_{m=0}^{\infty} \sum_{n=0}^{\infty} A_{m n} z^{m \bar{z}^{n}}
$$

is given we can express the coefficients $c_{m n}$ of $v_{s}(z)=\sum_{m=0}^{\infty} \sum_{n=0}^{\infty} c_{m n} s^{m} z^{n}$ in terms of the $A_{m 0}$.

Lemma 7.1. Let $\mathbf{E}(z, \bar{z}, t)$ be a generating function of the first kind, and let $u(z, \bar{z}) \in C+[\mathrm{E}(z, \bar{z}, t)]$ and $v_{s}=\mathcal{C}\left[\mathrm{E}^{(1)}(z, s, t)\right]$ be functions of different classes which jossess the same associate. Then

$$
c_{m n}=\sum_{\nu=0}^{n} \frac{A_{\nu 0} \Gamma(\nu+1)}{\pi^{1 / 2} \Gamma(\nu+1 / 2)} \int_{-1}^{1} E_{n-\nu}^{(m)}(t)\left(1-t^{2}\right)^{\nu-1 / 2} d t,
$$

where $\mathrm{E}_{\mu}^{(m)}(t)$ are the coefficients of the series development

$$
\mathrm{E}^{(1)}(z, s, t)=\sum_{m=0}^{\infty} \sum_{\mu=0}^{\infty} E_{\mu}^{(m)}(t) s^{m} z^{\mu} .
$$

Proof. Denoting by $f=\sum_{n=0}^{\infty} a_{n} z^{n}$ the R-associate of $u$ (see p. 312) we have by $(2.10)$

$$
a_{n}=1 / \tau_{n n} A_{n 0}, \quad \tau_{n n}=\pi^{1 / 2} \Gamma(n+1 / 2) / 2^{n} \Gamma(n+1)
$$

Thus

$$
\begin{aligned}
v_{s}(z) & =\int_{-1}^{1} \sum_{\mu=0}^{\infty} \sum_{m=0}^{\infty} E_{\mu}^{(m)}(t) s^{m} z^{\mu} \sum_{\nu=0}^{\infty} \frac{2^{\nu} \Gamma(\nu+1)}{\pi^{1 / 2} \Gamma(\nu+1 / 2)} A_{\nu 0} z^{\nu} \frac{\left(1-t^{2}\right)^{\nu-1 / 2}}{2^{\nu}} d t \\
& -\int_{-1}^{1} \sum_{n=0}^{\infty} \sum_{m=0}^{\infty} s^{m} z^{n}\left[\sum_{\nu=0}^{n} E_{n-\nu}^{(m)}(t) A_{\nu 0} \frac{\Gamma(\nu+1)}{\pi^{1 / 2} \Gamma(\nu+1 / 2)}\left(1-t^{2}\right)^{\nu-1 / 2}\right] d t,
\end{aligned}
$$

which yields (7.19).

If $\mathbf{E}(z, \bar{z}, t)$ is not a generating function of the first kind the only difference is that with the case considered above instead of (7.20) we obtain a more complicated expression representing the $a_{n}$ as functions of the $A_{m 0}$.

Writing now $C_{m n}=\left|c_{m n}\right|$, we obtain for the maximum $\mu(|s|, K|s|)$ of $\left|\sum_{m=0}^{\infty} \sum_{n=0}^{\infty} c_{m n} s^{m} z^{n}\right|$ on $E[|z|, K|s|]$, and sufficiently large $|s|$,

$$
\mu(|s|, K|s|) \leqq \exp \left(|s|^{\rho+\epsilon}\right), \quad \epsilon>0,
$$

where 
(7.23)

$$
\rho=\limsup _{m+n \rightarrow \infty} \frac{(m+n) \log (m+n)}{\log \left|1 / \sum_{\nu=0}^{n} \frac{A_{\nu 0} \Gamma(\nu+1)}{\pi^{1 / 2} \Gamma(\nu+1 / 2)} \int_{-1}^{1} E_{n-\nu}^{(m)}(t)\left(1-t^{2}\right)^{\nu-1 / 2} d t\right|}
$$

8. Upper bounds in terms of the coefficients $A_{n 0}$ of $u(z, \bar{z})$ $=\sum_{m=0}^{\infty} \sum_{n=0}^{\infty} A_{m n} z^{m} \bar{z}^{n}$ for functions, $n\left[s,(u(z, \bar{z})-b)^{-1}\right]$ and $I_{\lambda}[s,(u(z, \bar{z})$ $\left.-b)^{-1}\right]$, characteristic for the distribution of $b$-points of $u(z, \bar{z})$. We introduced in $\$ 6$ the function $v_{s}(z)$. In contrast to $\$ 7$ we shall now consider it as a function of one complex variable $z$, for some fixed real $s$.

Denoting $(1 / 2 \pi) \int_{0}^{2 \pi}\left[\log ^{+}\left|v_{s}\left(t e^{i \phi}\right)\right|\right] d \phi, t>0$, by $m\left[t, v_{s}(z)\right]$ we have for sufficiently large $s$

$$
m\left[K s, v_{s}(z)\right] \leqq \log \mu[s, K s] \leqq s^{\rho+\epsilon},
$$

where $\rho$ is determined by (7.24).

The fact that

$$
u(z, \bar{z})=v_{s}(z), \text { for }|z|=s,
$$

and therefore

$$
\begin{aligned}
n\left[s,(u(z, \bar{z})-b)^{-1}\right] & =n\left[s,\left(v_{s}(z)-b\right)^{-1}\right] \\
m\left[s,(u(z, \bar{z})-b)^{-1}\right] & =m\left[s,\left(v_{s}(z)-b\right)^{-1}\right],
\end{aligned}
$$

leads us to various conclusions on the distribution of the $b$-points of $u(z, \bar{z})$.

LEMMA 8.1. If $u(z, \bar{z}) \in \mathcal{C} \dagger(\mathrm{E})$ then

$$
n\left[s,(u(z, \bar{z})-b)^{-1}\right] \geqq 0, \bar{z} \text { conjugate to } z, s>0 \text {. }
$$

Proof. The inequality (8.4) follows from (6.2), (8.2) and the fact that $n\left[s,\left(v_{s}(z)-b\right)^{-1}\right] \geqq 0$.

Let $\mathfrak{g}^{1}(b)$ denote the totality of points of the positive $s$ axis, for which

$$
\left|\int_{-1}^{1}\left[\mathrm{E}^{(1)}(0, s, t) d t /\left(1-t^{2}\right)^{1 / 2}\right]-b\right| \geqq c, \quad c>0,
$$

$c$ being an arbitrary fixed constant.

Theorem 8.1. Let

$$
u(z, \bar{z})=\sum_{m=0}^{\infty} \sum_{n=0}^{\infty} A_{m n} z^{m} \bar{z}^{n}=\int_{-1}^{1} \mathrm{E}(z, \bar{z}, t) f\left(z\left(1-t^{2}\right) / 2\right) d t /\left(1-t^{2}\right)^{1 / 2},
$$

$\bar{z}$ conjugate to $z$, be an entire function of $x$ and $y$ which possesses only isolated $b$-points in the (real) $x y$-plane. Let further $\mathrm{E}^{(1)}(z, s, t)=\mathrm{E}\left(z, s^{2} / z, t\right)$ be an entire function of two complex variables $z$ and $s$. Then for every $\epsilon>0$ and for suffciently large $s, s \in \mathbb{B}^{1}(b / f(0))$, 


$$
\begin{gathered}
n\left[s,(u(z, \bar{z})-b)^{-1}\right] \leqq s^{\rho+\epsilon}, \\
m\left[s,(u(z, \bar{z})-b)^{-1}\right] \leqq s^{\rho+\epsilon},
\end{gathered}
$$

where $\rho$ is given by (7.24).

Proof. Applying classical techniques to the function

$$
v_{s}(z)=\int_{-1}^{1} \mathrm{E}^{(1)}(z, s, t) f\left(z\left(1-t^{2}\right) / 2\right) d t /\left(1-t^{2}\right)^{1 / 2}
$$

(see $[7$, p. 11]), we obtain

$$
\begin{aligned}
\log ^{+}\left|f(0) \int_{-1}^{1}\left[\mathrm{E}^{(1)}(0, s, t) d t /\left(1-t^{2}\right)^{1 / 2}\right]-b\right| & \\
& +m\left[r,\left(v_{s}(z)-b\right)^{-1}\right]+N\left[r,\left(v_{s}(z)-b\right)^{-1}\right] \\
= & m\left[r,\left(v_{s}(z)-b\right)\right] \log ^{+}\left|\frac{1}{f(0) \int_{-1}^{1}\left[\mathrm{E}^{(1)}(0, s, t) d t /\left(1-t^{2}\right)^{1 / 2}\right]-b}\right| \\
\leqq & m\left[r, v_{s}(z)\right]+\log ^{+}\left|\frac{1}{f(0) \int_{-1}^{1}\left[\mathrm{E}^{(1)}(0, s, t) d t /\left(1-t^{2}\right)^{1 / 2}\right]-b}\right| \\
& +2 \log ^{+} b+2 \log 2 .
\end{aligned}
$$

Substituting $r=K s, K>1$, noticing that

$$
m\left[K s, v_{s}(z)\right] \leqq \log \mu[s, K s],
$$

and using Theorem 7.1 we obtain

$$
\begin{aligned}
\log ^{+}\left|f(0) \int_{-1}^{1} \mathbf{E}^{(1)}(0, s, t) d t /\left(1-t^{2}\right)^{1 / 2}\right| & +m\left[K s,\left(v_{s}(z)-b\right)^{-1}\right] \\
& +N\left[K s,\left(v_{s}(z)-b\right)^{-1}\right] \leqq S^{\rho+\epsilon}+\log (1 / c)+O(1)
\end{aligned}
$$

for sufficiently large $s$ which belong to $\mathbb{B}^{1}(b / f(0))$. Since

$$
n\left[s,(u(z, \bar{z})-b)^{-1}\right]=n\left[s,\left(v_{s}(z)-b\right)^{-1}\right] \leqq \frac{N\left[K s,\left(v_{s}(z)-b\right)^{-1}\right]}{\log K}
$$

[see (8.3)], (8.9) implies (8.5).

Taking $|z|=s$ in (8.10) and using the second relation of (8.3) we obtain in similar manner inequality (8.6). $n\left[s,(u(z, \bar{z})-b)^{-1}\right]$ is a sectionally constant function of the real variable $s$ which in every interval $E\left[0<s \leqq S_{0}\right]$, $S_{0}<\infty$, possesses finitely many finite jumps.

We now introduce the expressions

$$
I_{\mu}(\rho)=\int^{\rho} \frac{d n\left[s,(u(z, \bar{z})-b)^{-1}\right]}{s^{\mu}}=\sum_{\left|z_{\nu}\right| \leqq_{\rho}} \frac{C\left[z_{\nu}(b)\right]}{\left|z_{\nu}(b)\right|^{\mu}}
$$


where $\int$ denotes the Stieltjes integral, $z_{\nu}(b)$ are the $b$-points of $u(z, \bar{z})$ and $C\left[z_{\nu}(b)\right]$ their indices.

LeMma 8.2. If

$$
n\left[s,(u(z, \bar{z})-b)^{-1}\right] \leqq s^{\lambda-\epsilon},
$$

then $I_{\lambda}(\infty)$ is bounded. Conversely, if $I_{\lambda}(\infty)$ is bounded, then

$$
\int^{\infty} \frac{n\left[s,(u(z, \bar{z})-b)^{-1}\right] d s}{s^{\lambda+1}}
$$

exists.

Proof. A well known procedure (see, for example, [7, p. 27]) yields immediately the lemma.

In fact, integrating by parts we obtain

$$
\begin{aligned}
\int_{r_{0}}^{r} \frac{n\left[s,(u(z, \bar{z})-b)^{-1}\right] d s}{s^{\lambda+1}}=\frac{n\left[r_{0},(u(z, \bar{z})-b)^{-1}\right]}{\lambda r_{0}^{\lambda}} \\
\quad-\frac{n\left[r,(u(z, \bar{z})-b)^{-1}\right]}{\lambda r^{\lambda}}+\frac{1}{\lambda} \int_{r_{0}}^{r} \frac{d n\left[s,(u(z, \bar{z})-b)^{-1}\right]}{s^{\lambda}} .
\end{aligned}
$$

Suppose now that (8.10) holds. Then it follows by (8.14) that $I_{\lambda}(r)$ $\leqq C_{1} r^{-\epsilon}+O(1)$, and therefore $I_{\lambda}(\infty)$ is bounded. Conversely $I_{\lambda}(\infty)<\infty$ implies that (8.13) is bounded since, by Lemma 8.1, $n\left[r,(u(z, \bar{z})-b)^{-1}\right]$ is nonnegative.

9. Some additional remarks concerning the value distribution of entire functions of the class $\mathcal{C}(\mathrm{E})$. In $\S \S 6-8$ we assumed that the generating function $\mathbf{E}(z, \bar{z}, t)$ has the property that $\mathbf{E}\left(z, s^{2} / z, t\right)$ is an entire function of $z$ and $s$. Clearly, in the general case $\mathbf{E}\left(z, s^{2} / z, t\right)=\sum_{m=0}^{\infty} \sum_{n=-\infty}^{\infty} H_{m n}(t) s^{m} z^{n}$ is not regular in $E[|s|<\infty, 0<|z|<\infty]$; in addition to singularities at $z=\infty$ and at $s=\infty$ it possesses an essential singularity at $z=0$. The application of our procedure will require the generalization of the corresponding results in the theory of entire functions of one and two complex variables to the case of functions which are singular also at $z=0$.

It will be of interest to indicate another method of attack for the study of value distribution of $u(z, \bar{z})$ in a domain bounded by an arbitrary closed curve, $\mathfrak{i}^{1}$.

In $[4, \S 5]$, see also footnote 18, p. 315 of the present paper, the operator

$$
u(z, \bar{z})=(2 \pi i)^{-1} \int f(Z) \mathrm{H}(z, \bar{z} ; Z) d Z,
$$

where the integral runs over $\mathrm{i}^{1}$, was introduced. Here $f$ is the $Q$-associate (see p. 312); 


$$
\mathrm{H}(z, \bar{z} ; Z)=\int_{-1}^{1}\left[\mathrm{E}(z, \bar{z}, t) /\left(Z-z\left(1-t^{2}\right) / 2\right)\right] d t /\left(1-t^{2}\right)^{1 / 2}
$$

is a solution of $\mathrm{L}(u)=0$ which possesses a singularity at the point $z=Z$, and $\mathfrak{i}^{1}$ is an arbitrary closed rectifiable curve which lies in the regularity domain of $f(Z)$ and includes in its interior the origin.

The representation $(9.1)$ of $u(z, \bar{z})$ is very suitable for investigation of $u(z, \bar{z})$ in the domain bounded by $i^{1}$. This method of attack leads to the study of the properties of the function $\mathrm{H}(z, \bar{z} ; Z)$.

In particular if certain differential relations between the coefficients of $\mathrm{L}(u)=0$ exist we obtain for $\mathbf{E}(z, \bar{z}, t)$ a closed expression in $t$ in terms of elementary functions (see $[3, \S 3]$ and $[5, \S 3]$ ), which is very convenient for investigations of this kind.

The formula (9.1) can also be used for the study of the derivatives of $u(z, \bar{z})$.

Lastly we observe that the introduction of the operator

$$
\int_{-1}^{1} \mathrm{E}(z, \bar{z}, t) f\left(z\left(1-t^{2}\right) / 2\right) d t /\left(1-t^{2}\right)^{1 / 2}
$$

enables us to investigate the behaviour of various subsequences,

$$
\sum_{m=\mu}^{M} \sum_{n=\nu}^{N} A_{m n} z^{m \bar{z}^{n}}
$$

of a function $u(z, \bar{z})=\sum_{m=0}^{\infty} \sum_{n=0}^{\infty} A_{m n} z^{m} \bar{z}^{n}$ of a class $\mathcal{C}(\mathbf{E})$.

Using the relation

$$
\begin{aligned}
A_{m n} z^{m} \bar{z}^{n}=(2 \pi i)^{-1} \int_{a} \int_{b} \int_{-1}^{1} \zeta_{1}^{-(m+1)} \zeta_{2}^{-(n+1)} \mathrm{E}\left(\zeta_{1} z, \zeta_{2} \bar{z}, t\right) \\
\cdot f\left(\zeta_{1} z\left(1-t^{2}\right) / 2\right) d \zeta_{1} d \zeta_{2} d t /\left(1-t^{2}\right)^{1 / 2}
\end{aligned}
$$

where $a$ and $b$ are rectifiable closed Jordan curves in the $\zeta_{1}$ and $\zeta_{2}$ planes, respectively, and employing standard methods of the theory of functions one may draw various conclusions about the left-hand side of (9.4) from the behaviour of $f$ and $\mathbf{E}$.

\section{BIBLIOGRAPHY}

1. Ludwig Baumgarten, Beiträge zur Theorie der ganzen Funktionen von zwei komplexen Veränderlichen, Monatshefte für Mathematik und Physik vol. 25 (1914) pp. 3-70.

2. Stefan Bergman, Über Kurvenintegrale von Funktionen zweier komplexen Veränderlichen, die Differentialgleichung $\Delta V+V=0$ befriedigen, Math. Zeit. vol. 32 (1930) pp. 386-406.

3. - Zur Theorie der Funktionen, die eine lineare partielle Differentialgleichung befriedigen, Rec. Math. (Mat. Sbornik) N.S. vol. 2 (1937) pp. 1169-1198.

4. - Linear operators in the theory of partial differential equations, Trans. Amer. Math. Soc. vol. 53 (1943) pp. 130-155. 
5. - The determination of some properties of a function satisfying a partial differential equation from its series development, Bull. Amer. Math. Soc. vol. 50 (1944) pp. 535-546.

6. - Solutions of partial differential equations of fourth order, Duke Math. J. vol. 11 (1944) pp. 617-649.

7. Rolf Nevanlinna, Theorème de Picard-Borel et la theorie des fonctions méromorphes, Paris, 1929.

8. Jules Sire, Sur les fonctions entières de deux variables d'ordre apparent total fini, Rend. Circ. Mat. Palermo vol. 31 (1910) pp. 1-91.

9. - Sur les fonctions entières de deux variables d'ordre apparent total fini et d̀ croissance réguliere par rapport d l'une des variab̈les, J. Math. Pures Appl. (6) vol. 9 (1913) pp. 1-37.

BROWN UNIVERSITY,

Providence, R. I. 